\title{
An analytical study of transport, mixing and chaos in an unsteady vortical flow
}

\author{
By V. ROM-KEDAR ${ }^{1}$, A. LEONARD ${ }^{2}$ AND S. WIGGINS \\ ${ }^{1}$ Applied Mathematics, California Institute of Technology, Pasadena, CA 91125, USA \\ ${ }^{2}$ Graduate Aeronautical Laboratories, California Institute of Technology, \\ Pasadena, CA 91125, USA \\ ${ }^{3}$ Applied Mechanics, California Institute of Technology, Pasadena, CA 91125, USA
}

(Received 2 May 1988)

We examine the transport properties of a particular two-dimensional, inviscid incompressible flow using dynamical systems techniques. The velocity field is time periodic and consists of the field induced by a vortex pair plus an oscillating strainrate field. In the absence of the strain-rate field the vortex pair moves with a constant velocity and carries with it a constant body of fluid. When the strain-rate field is added the picture changes dramatically; fluid is entrained and detrained from the neighbourhood of the vortices and chaotic particle motion occurs. We investigate the mechanism for this phenomenon and study the transport and mixing of fluid in this flow. Our work consists of both numerical and analytical studies. The analytical studies include the interpretation of the invariant manifolds as the underlying structure which govern the transport. For small values of strain-rate amplitude we use Melnikov's technique to investigate the behaviour of the manifolds as the parameters of the problem change and to prove the existence of a horseshoe map and thus the existence of chaotic particle paths in the flow. Using the Melnikov technique once more we develop an analytical estimate of the flux rate into and out of the vortex neighbourhood. We then develop a technique for determining the residence time distribution for fluid particles near the vortices that is valid for arbitrary strainrate amplitudes. The technique involves an understanding of the geometry of the tangling of the stable and unstable manifolds and results in a dramatic reduction in computational effort required for the determination of the residence time distributions. Additionally, we investigate the total stretch of material elements while they are in the vicinity of the vortex pair, using this quantity as a measure of the effect of the horseshoes on trajectories passing through this region. The numerical work verifies the analytical predictions regarding the structure of the invariant manifolds, the mechanism for entrainment and detrainment and the flux rate.

\section{Introduction}

In most fluid flows of interest, transport and mixing are dominated by convective processes so that the relative motions of fluid particles are all important. Unfortunately particle motion is generally more complex than the underlying fluid dynamics. For example, while the motion of three point vortices in an unbounded domain is integrable, particle motion in this flow can be chaotic (Aref 1983) and

$\dagger$ Present address: The James Franck Institute, The University of Chicago, Chicago, IL 60637, USA. 
certain simple steady, spatially periodic solutions to the Euler equations in three dimensions, known as ABC (Arnol'd, Beltrami and Childress) flows yield chaotic particle motion (Dombre et al. 1986).

Of course, if the fluid dynamics is sufficiently simple then particle motions are integrable and a direct analytical attack on the problem may be fruitful. An example in this class is the analysis of a diffusion flame by Marble (1985), involving the rolling up of an initially plane interface in the flow of a viscous line vortex in two dimensions. At the other end of the scale, when the flow is turbulent, direct numerical integration of the Navier-Stokes equations plus convective equations for passive scalars (Kerr 1985; Pope 1987) is a computational approach to mixing problems, whereas a theoretical approach might consist of constructing reasonable physical models for mixing processes (Broadwell 1989; Dimotakis 1989; Kerstein \& Ashurst 1984). In this paper we consider an intermediate case, one in which the flow is relatively simple but the particle motion is chaotic. We show that the recent rapid development in the theory of nonlinear dynamical systems and chaotic phenomena gives much hope for a rather extensive analysis of particle motion in such flows. Indeed, the dynamical systems approach to the study of fluid flows is very similar in spirit to the flow visualization techniques utilized in the experimental study of coherent structures in the sense that dynamical systems theory is concerned with the global topology of the flow from a Lagrangian point of view. Since to a good approximation temperature and mass move with the fluid velocity, understanding the structures governing particle motion in fluid flows is necessary for interpretations of flow visualizations (the visualization of motion of mass particles) and predictions of mass and heat transfer in technological applications.

The application of dynamical systems theory to the study of the global topology of fluid particle motions is not new. The first work appears to be that of Hénon (1966) who, acting on a suggestion of Arnol'd (1965), numerically studied the fluid particle motions in $\mathrm{ABC}$ flow. Hénon showed that the flow contained KAM tori as well as chaotic motions of the Smale horseshoe type. This flow has recently been the subject of more extensive study by Dombre et al. (1986). Chaotic particle motions in the ABC flows also have relevance to the kinematic dynamo problem, see Arnol'd \& Kortine (1983), Galloway \& Frisch (1986), and Moffatt \& Proctor (1984). Aref (1985) made the first explicit connection between particle motions in two-dimensional incompressible flow and two-dimensional Hamiltonian dynamical systems.

Since the study of fluid particle motions involves only kinematical considerations, the application, and hence, results of dynamical systems theory are independent of Reynolds number. For example, Aref \& Balachandar (1986) showed that unsteady Stokes flow between eccentric rotating cylinders, in which the rotation rate is modulated periodically in time, can exhibit chaotic particle motions of the Smale horseshoe type. Thus this particular Stokes flow is effectively non-reversible. This same flow has also been studied experimentally as well as theoretically by Chaiken et al. $(1986,1987)$. Ottino and coworkers (see Chien, Rising \& Ottino 1986; Khakar, Rising \& Ottino 1986; and Ottino et al. 1988) studied chaotic fluid particle motions in a variety of flows, both at small and large Reynolds numbers with particular emphasis on using dynamical systems techniques as a theoretical basis for the discussion of mixing processes. Broomhead \& Ryrie (1988) studied fluid particle motions in the velocity field of Taylor vortices close to the onset of the wavy instability and demonstrated the chaotic transference of fluid between neighbouring vortices. Feingold, Kadanoff \& Piro (1988) studied models for particle motion in 
three-dimensional time-dependent flows. Additional references on the application of dynamical systems techniques to the study of fluid particle trajectories are Suresh (1985) and Arter (1983).

In this paper we study fluid particle motion in the velocity field induced by two counter-rotating point vortices of equal strength subject to a time-periodic strain field. This is a fundamental type of flow which is relevant to a wide variety of applications as, for example, in the study of oscillatory flows in wavy-walled tubes (see Ralph 1986; Sobey 1985, and Appendix A), in the study of trailing vortices, and in the study of perturbed vortex rings (Shariff 1989).

The main difference between our analysis of the topology of a fluid flow via dynamical systems techniques and previous analyses is that, rather than just using the framework of dynamical systems theory to give a description of the topology and indicate the presence of chaotic fluid particle trajectories, we use the framework in order to calculate physically measurable quantities such as fluxes and the distribution of volumes via residence times. We do this by first identifying the structures in the flow responsible for these physical processes and then by using the dynamics of these structures to predict these physical quantities. Thus in some sense we realize the goal of the study of coherent structures for our problem. Additionally, in this paper we introduce two new concepts that play an important role in the study of mixing and transport processes due to chaotic fluid particle motions.

The first is Tangle Dynamics. In $\$ 3$ we review how the study of particle motions in two-dimensional incompressible time-periodic fluid flows can be reduced to the study of a two-dimensional map. It is well known in the dynamical systems literature that such maps may possess resonance bands consisting of alternating hyperbolic and elliptic periodic points. This has fluid dynamical significance in the sense that the stable and unstable manifolds of the hyperbolic points create partial barriers to transport in the flow. Additionally, these stable and unstable manifolds may intersect many times resulting in a complicated geometrical structure that dramatically influences the stretching and deformation of fluid elements. We develop analytical and computational techniques which we refer to as tangle dynamics that allow us to compute the rate of transport of fluid between regions separated by these partial barriers. From this information we can compute residence time distributions and, more generally, determine the effect of a resonance band on a fluid element. We develop these ideas in the context of the specific flow considered in this paper; however, recently the methods have been generalized to apply to any twodimensional time-periodic fluid flow, see Rom-Kedar \& Wiggins (1989). These techniques are mathematically exact and represent a fundamental improvement over the approximate phase-space transport models of MacKay, Meiss \& Percival (1984). These models rely on the assumption of an infinite diffusion rate within the chaotic regions. Such an assumption is not applicable to the flow that we are studying.

The second new concept is Finite Time Stretch. Ottino (1988) has shown the relationship between the notion of a Liapunov exponent from dynamical systems theory and the stretching of fluid elements. However, the Liapunov exponent is a quantity computed for a single fluid particle trajectory which is time averaged in an asymptotic sense. Thus there is a practical limitation of this quantity in that, for many open flows, most fluid particles spend only a finite time in the chaotic zone rendering the classical theory of Liapunov exponents inappropriate. This is so because the asymptotic time average for such trajectories would give a zero 
exponent. However, using tangle dynamics and proof of the existence of chaotic particle motions, we are able to determine which particles should experience temporary exponential stretching and the finite time interval over which most of this stretching will take place. We then quantify the stretching by considering the total stretch suffered during this finite period of time.

This paper is organized as follows: In $\$ 2$ we derive the veloeity field for the oscillating vortex pair and in $\S 3$ we begin our analysis of the velocity field by introducing the Poincaré map. In $\$ 4$ we discuss three qualitatively distinct regions which arise in our flow: the free flow region, the core, and the mixing region. We discuss tangle dynamics and the associated mechanism for mass transport in the flow in $\$ 5$ and we consider mass transport in detail and give precise definitions to the concepts of entrainment and detrainment in terms of tangle dynamics in $\$ 6$, along with the results of numerical computations. In $\S 7$ we discuss the concept of chaos and show how it arises in our flow, and in $\S 8$ we discuss mixing and the total stretch of fluid elements as they pass through regions containing localized chaos. Summary and conclusions are given in $\$ 9$.

\section{Oscillating vortex pair}

We examine the flow governed by a vortex pair in the presence of an oscillating external strain-rate field. The vortices have circulations $\pm \Gamma$ and are separated by a nominal distance $2 d$ in the $y$-direction. The stream function for the flow in a frame moving with the average velocity of the vortices is

$$
\Psi=-\frac{\Gamma}{4 \pi} \log \left[\frac{\left(x-x_{\mathrm{v}}\right)^{2}+\left(y-y_{\mathrm{v}}\right)^{2}}{\left(x-x_{\mathrm{v}}\right)^{2}+\left(y+y_{\mathrm{v}}\right)^{2}}\right]-V_{\mathrm{v}} y+\epsilon x y \sin (\omega t),
$$

where $\left(x_{\mathrm{v}}(t), \pm y_{\mathrm{v}}(t)\right)$ are the vortex positions, $\epsilon$ is the strain rate and $V_{\mathrm{v}}$ is the average velocity of the vortex pair. If $\epsilon=0$ then $\left(x_{\mathrm{v}}, y_{\mathrm{v}}\right)=(0, d)$ and $V_{v}=\Gamma / 4 \pi d$. The equations of particle motion are

$$
\frac{\partial x}{\mathrm{~d} t}=\frac{\partial \Psi}{\partial y}, \quad \frac{\mathrm{dy}}{\mathrm{d} t}=-\frac{\partial \Psi}{\partial x} .
$$

We show, as an example, in Appendix A that this flow approximates the flow induced by a vortex pair in a wavy-walled channel. We obtain dimensionless variables as follows:

$$
\frac{x}{d} \rightarrow x, \quad \frac{y}{d} \rightarrow y, \quad \frac{\Gamma t}{2 \pi d^{2}} \rightarrow t, \quad \frac{\epsilon}{\omega} \rightarrow \epsilon, \quad \frac{2 \pi d V_{\mathrm{v}}}{\Gamma} \rightarrow \nu_{\mathrm{v}}, \quad \frac{\Gamma}{2 \pi \omega d^{2}} \rightarrow \gamma, \quad \frac{2 \pi \Psi}{\Gamma} \rightarrow \Psi .
$$

Then (2.1) and (2.2) become

$$
\begin{aligned}
& \frac{\mathrm{d} x}{\mathrm{~d} t}=-\left[\frac{\left(y-y_{\mathrm{v}}\right)}{\left(x-x_{\mathrm{v}}\right)^{2}+\left(y-y_{\mathrm{v}}\right)^{2}}-\frac{\left(y+y_{\mathrm{v}}\right)}{\left(x-x_{\mathrm{v}}\right)^{2}+\left(y+y_{\mathrm{v}}\right)^{2}}\right]-\nu_{\mathrm{v}}+\frac{\epsilon x}{\gamma} \sin (t / \gamma), \\
& \frac{\mathrm{dy}}{\mathrm{d} t}=\left(x-x_{\mathrm{v}}\right)\left[\frac{1}{\left(x-x_{\mathrm{v}}\right)^{2}+\left(y-y_{\mathrm{v}}\right)^{2}}-\frac{1}{\left(x-x_{\mathrm{v}}\right)^{2}+\left(y+y_{\mathrm{v}}\right)^{2}}\right]-\frac{\epsilon y}{\gamma} \sin (t / \gamma) .
\end{aligned}
$$

Using the fact that a point vortex is convected with the flow but does not induce selfvelocity we obtain the following equations for the vortex position locations:

$$
\frac{\mathrm{d} x_{\mathrm{v}}}{\mathrm{d} t}=\frac{1}{2 y_{\mathrm{v}}}-\nu_{\mathrm{v}}+\frac{\epsilon x_{\mathrm{v}}}{\gamma} \sin (t / \gamma), \quad \frac{\mathrm{d} y_{\mathrm{v}}}{\mathrm{d} t}=-\frac{\epsilon y_{\mathrm{v}}}{\gamma} \sin (t / \gamma)
$$




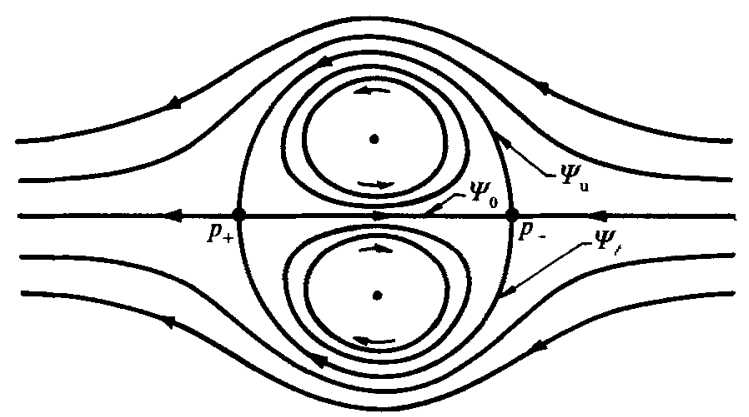

FIGURE 1. Streamlines of the unperturbed flow.

The resulting motion of the vortices is relatively simple. Equations (2.4) with the initial conditions $x_{\mathrm{v}}(0)=0, y_{\mathrm{v}}(0)=1$, are easily integrated to give

$$
x_{\mathrm{v}}(t)=\frac{1}{2} \gamma \mathrm{e}^{-\epsilon(\cos (t / \gamma)-1)} \int_{0}^{t / \gamma}\left[1-2 v_{\mathrm{v}} \mathrm{e}^{\varepsilon(\cos (s)-1)}\right] \mathrm{d} s, \quad y_{\mathrm{v}}(t)=\mathrm{e}^{\epsilon(\cos (t / \gamma)-1)} \cdot(2.5 a, b)
$$

The requirement that the mean velocity of the vortex pair be zero in the moving frame yields $\nu_{\mathrm{v}}=\mathrm{e}^{\epsilon} / 2 I_{0}(\epsilon)$, where $I_{0}$ is the modified Bessel function of order zero. From (2.5) it is clear that the vortices oscillate in orbits near the points $(0, \pm 1)$. Thus we term the resulting flow given by (2.3) the oscillating vortex pair (OVP) flow.

Equations (2.3) together with (2.5) give the equations of particle motion as a function of two dimensionless parameters $\gamma$ and $\epsilon$, proportional to vortex strength and strain rate, respectively. For most of the analysis that follows $\epsilon$ can take on arbitrary values. However, for the perturbation calculations we shall assume that $\epsilon$ is small and will require an expansion of the right-hand side of (2.3) in powers of $\epsilon$. This expansion yields equations of motion for fluid particles which are of the form of a periodically perturbed integrable Hamiltonian system:

$$
\begin{aligned}
& \frac{\mathrm{d} x}{\mathrm{~d} t}=f_{1}(x, y)+\epsilon g_{1}(x, y, t / \gamma ; \gamma)+O\left(\epsilon^{2}\right) \\
& \frac{\mathrm{d} y}{\mathrm{~d} t}=f_{2}(x, y)+\epsilon g_{2}(x, y, t / \gamma ; \gamma)+O\left(\epsilon^{2}\right)
\end{aligned}
$$

The functions $f_{i}, g_{i}$ are given in Appendix B.

For $\epsilon=0$ the phase portrait of the integrable Hamiltonian system, or equivalently the streamlines of the flow induced by a vortex pair in the frame moving with the vortices, appears in figure 1 . Note that for this case, there are two hyperbolic stagnation points $p_{-}, p_{+}$connected by three limiting streamlines $\Psi_{\mathrm{u}}, \Psi_{0}$ and $\Psi_{\ell}$ defined by $\left.\Psi(x, y)\right|_{\epsilon \rightarrow 0}=0,|x| \leqslant \sqrt{ } 3$, with $y>0, y=0$, and $y<0$ respectively. Thus a fixed, closed volume of fluid or 'bubble' is bounded by the limiting streamlines and moves with the vortex pair for all times. As we shall see below, this picture changes dramatically when $\epsilon \neq 0$. Note also that, for any $\epsilon$, the flow is symmetric about the $x$-axis and thus we need only study the flow in the upper half-plane. Such symmetry would be present in axisymmetric flows. If the strain-rate field is not aligned with the $(x, y)$-axes the straight line connecting the two vortices also rotates periodically, but the qualitative behaviour of the particle motion is the same as that discussed in the following but with the added complication of transport between the upper and lower half-planes. 


\section{Analysis: the Poincaré map}

We are interested in the structure of the flow generated by the velocity field (2.3) and how the structure varies as the parameters $\gamma$ and $\epsilon$ are varied. A brute force method for achieving this goal would be to numerically integrate (2.3) for a large number of initial conditions for the range of $\gamma$ and $\epsilon$ values of interest. Although this would be an efficient means for generating a large list of numbers, it is not at all clear how one would extract information concerning the structure of the flow from this list. One might try plotting the trajectories of a large number of fluid particles in space; however, because the velocity field is unsteady, these trajectories may intersect themselves as well as others many times leading to a complicated topological structure which might obscure relatively simple structures whose dynamics essentially govern the flow. In order to better understand the dynamics of the unsteady flow generated by (2.3) we will study the associated Poincaré map.

Roughly speaking, the Poincaré map of the flow is constructed by associating to a fluid particle at a fixed phase of the external strain-rate field its location under evolution by the flow after one period of the strain-rate field. More mathematically, we rewrite the unsteady two-dimensional velocity field (2.3) as a steady threedimensional velocity field by introducing the phase of the strain-rate as a new dependent variable. We do this by defining the function

$$
\theta(t)=t / \gamma \quad \bmod 2 \pi
$$

in which case (2.3) and (2.5) can be written as

$$
\dot{x}=\frac{\partial \Psi}{\partial y}(x, y, \theta ; \epsilon, \gamma), \quad \dot{y}=-\frac{\partial \Psi}{\partial x}(x, y, \theta ; \epsilon, \gamma), \quad \dot{\theta}=1 / \gamma,
$$

and for small $\epsilon(2.6)$ becomes

$$
\dot{x}=f_{1}(x, y)+\epsilon g_{1}(x, y, \theta ; \gamma)+O\left(\epsilon^{2}\right), \quad \dot{y}=f_{2}(x, y)+\epsilon g_{2}(x, y, \theta ; \gamma)+O\left(\epsilon^{2}\right), \quad \dot{\theta}=1 / \gamma
$$

A two-dimensional cross-section of the three-dimensional phase space of (3.1) or (3.2) is given by

$$
\Sigma^{\bar{\theta}}=\{(x, y, \theta) \mid \theta=\bar{\theta} \in(0,2 \pi]\},
$$

and the Poincaré map of $\Sigma^{\bar{\theta}}$ into $\Sigma^{\bar{\theta}}$ is defined as

$$
\begin{gathered}
T_{\bar{\theta}}: \Sigma^{\bar{\theta}} \rightarrow \Sigma^{\bar{\theta}} \\
(x(\bar{\theta}), y(\bar{\theta})) \mapsto(x(\bar{\theta}+2 \pi), y(\bar{\theta}+2 \pi)) .
\end{gathered}
$$

So studying the flow via the Poincare map is equivalent to sampling fluid particle trajectories at time intervals equal to the period of the strain-rate field.

The main advantage obtained from using the Poincaré map to study unsteady, time-periodic velocity fields is that the technique tends to filter out redundant dynamical phenomena and reveal the underlying structures which govern various properties of the flow such as mixing and transport. For example, a periodic particle trajectory in the flow which may have a very complicated topological structure is manifested as a finite, discrete set of points for the Poincaré map. Many more examples will follow throughout the rest of the paper. Also, in Appendix $E$ we collect several useful properties of Poincaré maps.

Our goal is to study properties of the orbit structure of the Poincare map in order to discover the structures necessary to predict mixing and transport properties of the flow. 


\section{Three qualitatively distinct flow regions}

Let us recall the structure of the unperturbed velocity field now in the context of the Poincare map. In this case the velocity field is steady and fluid particles follow the streamlines defined by the level curves of the stream function. Thus orbits of the Poincaré maps are sequences of discrete points lying on the streamlines. The streamlines are examples of invariant curves or manifolds of the Poincare map, meaning that particles which start on such a curve must thereafter remain on that curve under all iterations of the Poincaré map. The stagnation points $p_{-}$and $p_{+}$are fixed points of the Poincaré map. Orbits of fluid particles on $\Psi_{\mathrm{u}}$, and $\Psi_{\ell}$ approach $p_{+}$ asymptotically in positive time and $p_{-}$asymptotically in negative time. In the terminology of dynamical systems theory, $\Psi_{\mathrm{u}}$ and $\Psi_{\ell}$ are referred to as the stable manifold of $p_{+}$, denoted $W_{+}^{\mathrm{s}}$; and $\Psi_{0}$ plus $\left(-\infty, p_{+}\right)$is the unstable manifold of $p_{+}$, denoted $W_{+}^{\mathrm{u}}$. Similarly, $\Psi_{\mathrm{u}}$ and $\Psi_{\ell}$ are referred to as the unstable manifold of $p_{-}$, denoted $W_{-}^{\mathrm{u}}$; and $\Psi_{0}$ plus $\left(p_{-}, \infty\right)$ is the stable manifold of $p_{-}$, denoted $W_{-}^{\mathrm{s}}$. Orbits of fluid particles starting on $\Psi_{u}, \Psi_{0}$, and $\Psi_{\ell}$ are referred to as heteroclinic orbits and $\Psi_{\mathrm{u}} \cup \Psi_{0} \cup\left\{p_{+}\right\} \cup\left\{p_{-}\right\}$and $\Psi_{\ell} \cup \Psi_{0} \cup\left\{p_{+}\right\} \cup\left\{p_{-}\right\}$are said to form heteroclinic cycles. For brevity we shall simply refer to the closed curve $\Psi_{\mathrm{u}} \cup \Psi_{\ell} \cup\left\{p_{+}\right\} \cup\left\{p_{-}\right\}$as the limiting streamlines.

Notice that fluid particle motions outside the region bounded by the limiting streamlines are qualitatively different than those inside this region. We now want to discuss more fully the different possible fluid particle motions and how they are constrained by structures in the flow.

\subsection{The free flow region}

Under the influence of the unperturbed velocity field fluid particles outside the region bounded by the limiting streamlines move from right to left along the unbounded streamlines. We refer to this as the free flow region. Under the influence of the externally strained velocity field fluid particles which are sufficiently far from the limiting streamlines behave in the same manner as those in the unperturbed velocity field. Particles move from right to left as before now with vertical oscillations but their trajectories never encircle a vortex.

\subsection{The core}

We refer to the region of fluid which is permanently trapped and hence moves with the vortex pair for all time as the core. For the unperturbed flow the core is the fluid within the limiting streamlines and it contains two separate cells with boundaries $C_{\mathrm{u}}=\Psi_{\mathrm{u}} \cup \Psi_{0} \cup\left\{p_{+}\right\} \cup\left\{p_{-}\right\}$and $C_{\ell}=\Psi_{\ell} \cup\left\{p_{+}\right\} \cup\left\{p_{-}\right\}$. Fluid particles in the interior of $C_{\mathrm{u}}$ and $C_{\ell}$ move in closed paths along the streamlines of the unperturbed velocity field. We can uniquely label each closed streamline in $C_{\mathrm{u}}$ and $C_{\ell}$ by the area which it encloses. We denote this area by $I$ (note: in the context of Hamiltonian mechanics $I$ is called the action, see Arnol'd 1978). Associated with each closed streamline is a period $T(I)$ which is the time needed for a particle starting on the streamline to make one complete circuit along the streamline. The period goes to zero as the point vortices are approached and to infinity as $C_{\mathrm{u}}$ and $C_{\ell}$ are approached.

We now interpret this extremely simple motion of fluid particles in the unperturbed case inside $C_{\mathrm{u}}$ and $C_{\ell}$ in terms of orbits of the Poincaré map. The streamlines in this case are examples of invariant curves of the Poincare map. That is, orbits of fluid particles which start on the streamlines must always stay on the streamlines. There are two types of orbits depending on whether or not the number $T(I) / 2 \pi \gamma$ is rational 


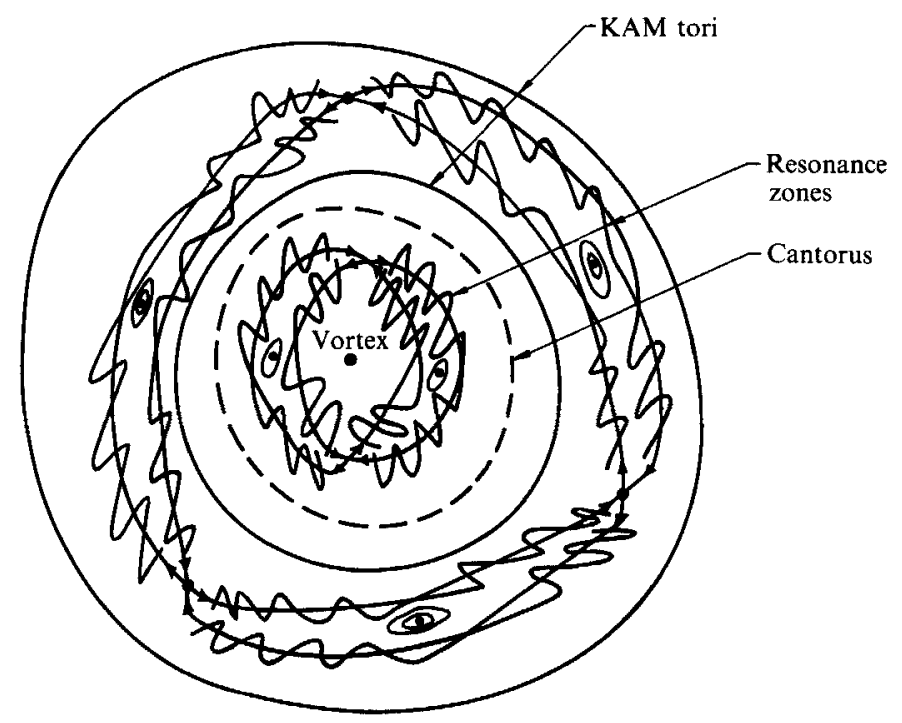

FIQURE 2. Resonance bands and KAM tori.

or irrational (note : in the context of dynamical systems theory the number $T(I) / 2 \pi \gamma$ is referred to as a rotation number). These two types of orbits behave very differently under the influence of the time-periodic strain-rate field.

$T(I) / 2 \pi \gamma=p / q: p, q$, integers. In this case every fluid particle on the invariant circle in the Poincare map in the unperturbed case returns to its original position after $q$ cycles of the Poincaré map. However, in the process it makes $p$ complete revolutions around the invariant circle. Thus all fluid particles on the invariant circle move periodically with period $q$.

In general, this situation can be expected to change dramatically under the influence of the time-periodic strain-rate field. The invariant circle is destroyed and a finite (usually even) number of periodic orbits of alternating stability type will be preserved in the externally strained case. Half of the periodic orbits will be stable and half will be unstable of saddle type. The stable and unstable manifolds of the saddletype motions may intersect transversely, yielding chaotic fluid particle motions. The resulting structure is known as a $p / q$ resonance band or stochastic layer, see Arnol'd \& Avez (1968) for more details.

$T(I) / 2 \pi \gamma=\omega: \omega$ irrational. In the unperturbed velocity field every fluid particle starting on an invariant circle of the Poincaré map rotates around the circle, never returning to its initial position. Two possibilities for the behaviour of these orbits under external strain are as follows:

$K A M$ tori. If $\omega$ is sufficiently poorly approximated by rational numbers, i.e. it satisfies a diophantine condition (see Arnol'd \& Avez 1968 or Moser 1973), then, for sufficiently small-amplitude strain rates $\epsilon$, the invariant circle is preserved in the perturbed Poincaré map. This invariant circle is referred to as a $K A M$ torus after Kolmogorov, Arnol'd and Moser who first proved the result (known as the KAM theorem). KAM tori are extremely important since they represent total barriers to fluid motion and hence strongly influence transport.

Cantori. If $\omega$ fails to satisfy the number theoretic hypotheses of the KAM theorem then the work of Percival (1980), Aubry \& Le Daeron (1983), and Mather (1984) implies that the invariant circle may break down under the time-periodic strain-rate 
field into an invariant cantor set or cantorus. The dynamics on the cantorus are similar to the dynamics on the KAM torus. However, the cantorus contains gaps which permit the (possibly very slow) passage of fluid.

We refer the reader to figure 2 for an illustration of resonance bands, KAM tori, and cantori in the core. Our primary concern is with particle motion in the mixing region defined below. Thus we only remark that these three structures govern the fluid transport within the core. In the parameter range we are studying the largest KAM torus exists, which serves as a complete barrier to the flow, and therefore will prevent the mixing of outer fluid with the fluid in the core. An interesting question is whether the core region is composed of a single region bounded by a KAM torus or if there are islands outside the largest KAM torus in which the motion is bounded. During the numerical experiments, described in $\$ 6$, we observed that for $\gamma=0.5$ there is only one observable core region while for $\gamma=1.38$ there seem to be at least two (see figure 20 for an indication).

\subsection{The mixing region}

We now want to focus our attention on $p_{+}$And $p_{-}$and their stable and unstable manifolds. In the unperturbed flow $W_{+}^{\mathrm{s}}$ and $W_{-}^{\mathrm{u}}$ coincide along the streamlines $\Psi_{\mathrm{u}}$ and $\Psi_{\ell}$ to create a boundary separating the core from the free flow region. With the addition of external strain we can make the following assertions : (i) For sufficiently small-amplitude strain fields (i.e. for $\epsilon$ sufficiently small) $p_{+}$and $p_{-}$persist as fixed points of the Poincaré map. We shall denote them by $p_{+, \epsilon}$ and $p_{-, \epsilon}$, respectively. (ii) The stable and unstable manifolds of $p_{+}$and $p_{-}$persist to become the stable and unstable manifolds of $p_{+, \varepsilon}$ and $p_{-, \epsilon}$. We denote them by $W_{+, \varepsilon}^{\mathrm{s}}, W_{+, \epsilon}^{\mathrm{u}}, W_{-, \varepsilon}^{\mathrm{s}}$, and $W_{-, \epsilon}^{\mathrm{u}}$, respectively. The two results follow from general theorems regarding the persistence of invariant manifolds which can be found in Fenichel (1971) or Hirsch, Pugh \& Shub (1977) and they are independent of the specific analytical form of the time-periodic strain-rate field (note: these results would also apply to quasi-periodic strain rates, see Wiggins, 1987, 1988).

We shall see in $\S 6$ that for arbitrary $\epsilon$, (i) persistence of invariant manifolds for arbitrary values may be decided by computation, (ii) particle transport is governed by the invariant manifolds, and (iii) that the unstable manifold is the observable structure in a broad class of flow visualizations.

From our discussion of the symmetry of the velocity field in $\$ 3$, it follows that $y=0$ is always an invariant manifold for both the perturbed and unperturbed velocity field. This implies that $\Psi_{0}=W_{+}^{u} \cup W_{-}^{\mathrm{s}}=W_{+, \varepsilon}^{\mathrm{u}} \cup W_{-, \varepsilon}^{\mathrm{s}}$ persists as an invariant streamline. However, the interpretation of $W_{+, \epsilon}^{\mathrm{s}}$ and $W_{-, \epsilon}^{\mathrm{u}}$ is more subtle since they need not coincide as in the unperturbed case. Now $W_{+, \epsilon}^{\mathrm{s}}$ and $W_{-, \epsilon}^{\mathrm{u}}$ are smooth invariant curves and a fluid particle path starting on these curves in the continuous time flow is represented as an infinite set of discrete points on these curves in the Poincaré section. As such it is possible for $W_{-, \epsilon}^{u}$ and $W_{+, \epsilon}^{s}$ to intersect in an isolated point as shown in figure 3 . Note that figure 3 could not hold in the unperturbed case because, for steady flows, particle paths must coincide with streamlines and streamlines cannot intersect in isolated non-stagnation points without violating uniqueness of solutions of ordinary differential equations. If we consider the orbit of this point of intersection under the Poincaré map, then by invariance of $W_{-, \epsilon}^{\mathrm{u}}$ and $W_{+, \epsilon}^{\mathrm{s}}$ it must forever remain on both $W_{-, \epsilon}^{u}$ and $W_{+, c}^{\mathrm{s}}$ resulting in a geometrical shape similar to that shown in figure 3 . This splitting of the stable manifold of $p_{+}$and the unstable

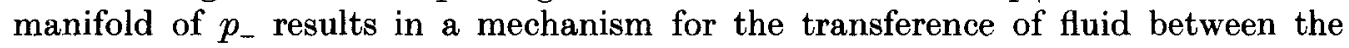
vicinity of the core and the vicinity of the free flow region. It also provides the 


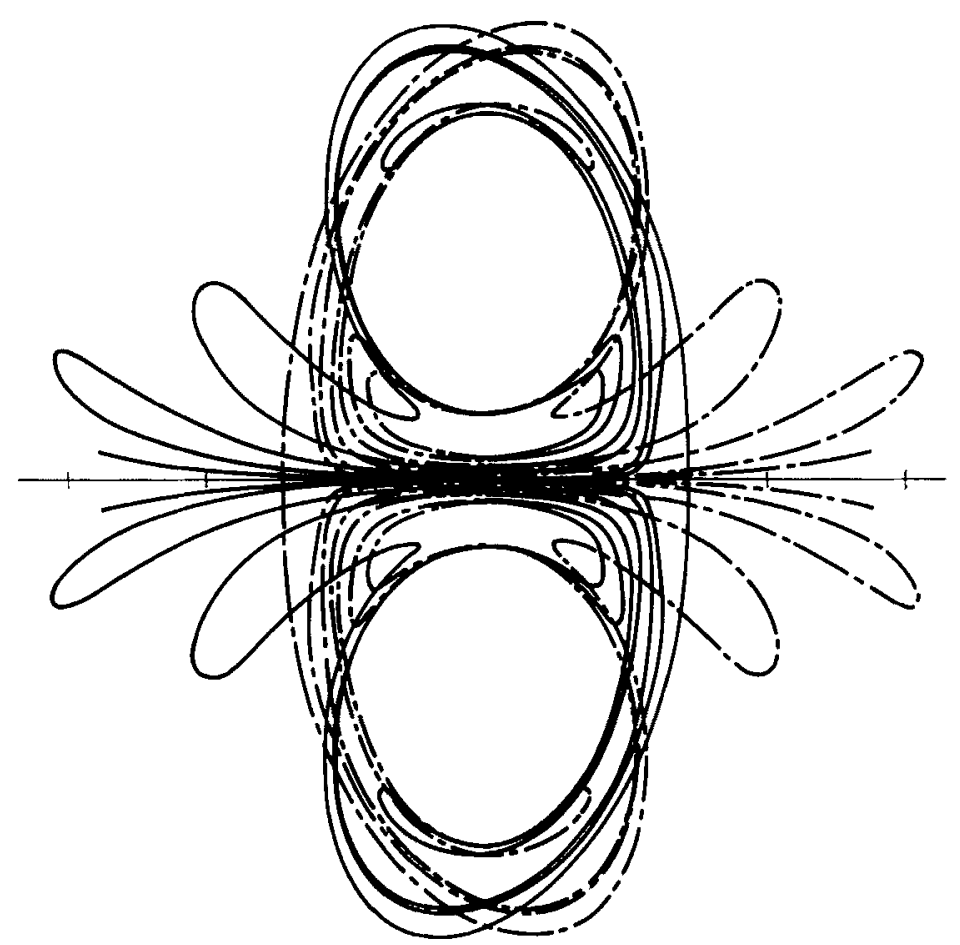

Figure 3. The homoclinic tangle in the mixing region. - Unstable manifold; - - , stable manifold.

mechanism for chaotic particle motion. For this reason, we refer to the region bounded roughly by the envelopes of $W_{-, \epsilon}^{\mathrm{u}}$ and $W_{+, \epsilon}^{\mathrm{s}}$ as the mixing region. A precise definition of the mixing region is given in $\$ 6$. The mixing region, of course, does not exist for the unperturbed case. One characterization that distinguishes the free flow region, the mixing region, and the core is that they consist of particle trajectories that encircle a vortex zero, a finite, and an infinite number of times, respectively.

\section{The Melnikov technique}

An analytical technique which allows us to predict the behaviour of $W_{+, \epsilon}^{\mathrm{s}}$ and $W_{-, \epsilon}^{\mathrm{u}}$ for small $\epsilon$ was developed by Melnikov (1963) and consist of a measurement of the distance between $W_{+, \varepsilon}^{\mathrm{s}}$ and $W_{-, \epsilon}^{\mathrm{u}}$. Up to a known normalization factor, the first-order term of the Taylor series expansion about $\epsilon=0$ of the distance between $W_{+, \epsilon}^{\text {s }}$ and $W_{-, e}^{\text {u }}$ can be computed without solving (2.3) explicitly. This first-order term is known as the Melnikov function. In Appendix C, we discuss the geometry of the Melnikov function as well as some of the relevant technical points behind its derivation. In this section, we state the results of the calculations for our problem.

The distance between $W_{+, \varepsilon}^{\mathrm{s}}$ and $W_{-, \epsilon}^{\mathrm{u}}$ is given by

$$
d\left(t_{0}, \epsilon\right)=\epsilon \frac{M\left(t_{0}\right)}{\left\|f\left(q_{\mathrm{u}}\left(-t_{0}\right)\right)\right\|}+O\left(\epsilon^{2}\right),
$$

where $\boldsymbol{q}_{\mathrm{u}}(t)$ is a heteroclinic fluid particle trajectory of the unperturbed velocity field lying in $\Psi_{\mathrm{u}}, t_{0}$ parametrizes distance along $\Psi_{\mathrm{u}}$, and

$$
\left\|f\left(q_{\mathrm{u}}\left(-t_{0}\right)\right)\right\|=\left[\left(f_{1}\left(q_{\mathrm{u}}\left(-t_{0}\right)\right)\right)^{2}+\left(f_{2}\left(\boldsymbol{q}_{\mathrm{u}}\left(-t_{0}\right)\right)\right)^{2}\right]^{\frac{1}{2}} .
$$




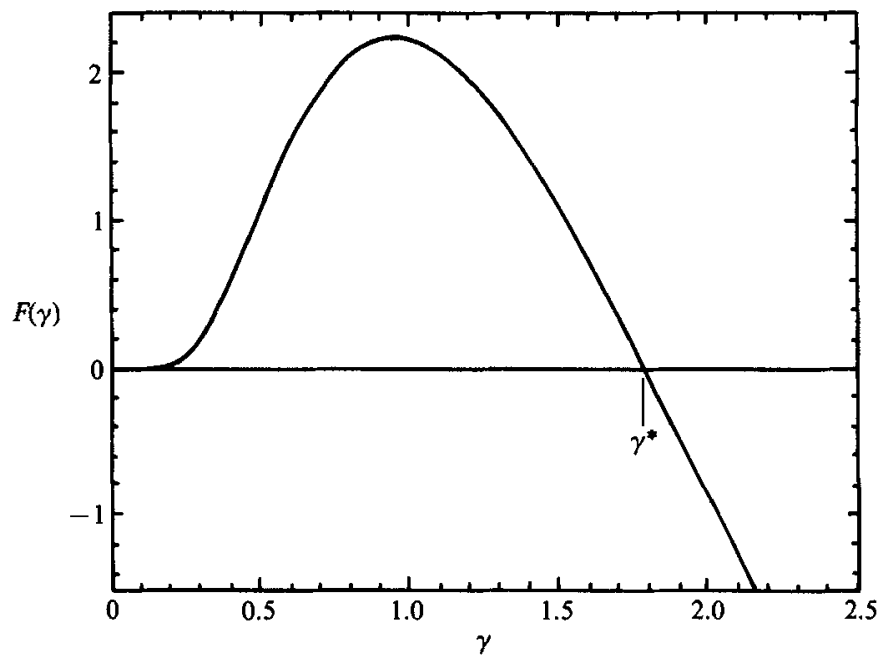

Figure 4. Graph of $F(\gamma)$.

The Melnikov function $M\left(t_{0}\right)$ is defined to be

$$
M\left(t_{0}\right)=\int_{-\infty}^{\infty}\left[f_{1}\left(\boldsymbol{q}_{\mathrm{u}}(t)\right) g_{2}\left(\boldsymbol{q}_{\mathrm{u}}(t), t+t_{0}\right)-f_{2}\left(\boldsymbol{q}_{\mathrm{u}}(t)\right) g_{1}\left(\boldsymbol{q}_{\mathrm{u}}(t), t+t_{0}\right)\right] \mathrm{d} t,
$$

and Melnikov's theorem (see Appendix C) shows us that simple zeros of $M\left(t_{0}\right)$ (i.e. $M\left(t_{0}\right)=0, \partial M / \partial t_{0} \neq 0$ ) imply simple zeros of $d\left(t_{0}, \epsilon\right)$ for $\epsilon$ sufficiently small. We remark that $d\left(t_{0}, \epsilon\right)$ may be either positive or negative as it is actually the signed distance between $W_{+, \varepsilon}^{s}$ and $W_{-, \epsilon}^{u}$. In Appendix $C$, we show that the sign of $d\left(t_{0}, \epsilon\right)$ gives us information concerning the relative orientation of $W_{+, \epsilon}^{\mathrm{s}}$ and $W_{-, \epsilon}^{\mathrm{u}}$. Also note that $\left\|f\left(q_{\mathrm{u}}\left(-t_{0}\right)\right)\right\| \rightarrow 0$ exponentially fast as $t_{0} \rightarrow \pm \infty$ which implies that $\left|d\left(t_{0}, \epsilon\right)\right| \rightarrow \infty$ as $t_{0} \rightarrow \pm \infty$. This just reflects the fact that $W_{+, \epsilon}^{\mathrm{s}}$ and $W_{-, \epsilon}^{\mathrm{u}}$ oscillate unboundedly near $p_{-, \epsilon}$ and $p_{+, \epsilon}$, respectively.

We numerically calculate the Melnikov function for the velocity field (2.3) and obtain

$$
M\left(t_{0}\right)=\frac{F(\gamma)}{\gamma} \sin \left(t_{0} / \gamma\right)
$$

where $F(\gamma)$ is plotted in figure 4 . Note that for fixed $\gamma, M\left(t_{0}\right)$ has an infinite number of isolated zeros at which $\partial M / \partial t_{0} \neq 0$. As discussed in Appendix $\mathrm{C}$, these correspond to transverse intersections of $W_{+, \varepsilon}^{\mathrm{s}}$ and $W_{-, \epsilon}^{\mathrm{u}}$ and therefore we obtain a direct analytical confirmation of figure 3 . At $\gamma \approx 1.78, F(\gamma)$ changes sign, which corresponds to a change in the orientation of the intersection of $W_{+, \epsilon}^{\mathrm{s}}$ and $W_{-, \epsilon}^{\mathrm{u}}$. For $\gamma \approx 1.78, M\left(t_{0}\right)=0$ implying that $d\left(t_{0}, \epsilon\right)=O\left(\epsilon^{2}\right)$. In figure 5 we present the manifolds computed numerically for several values of $\gamma$ confirming the change of orientation of the intersection.

\section{Tangle dynamics}

We now describe the dynamics associated with the tangling of the stable and unstable manifolds of $p_{+, \epsilon}$ and $p_{-, \epsilon}$. Specifically, we shall describe the essential dynamical mechanisms for fluid transport within the mixing region. We shall see that the properties of invariance of the stable and unstable manifolds as well as the orientation preserving property of the Poincaré map render a temporal simplicity to 
V. Rom-Kedar, A. Leonard and S. Wiggins
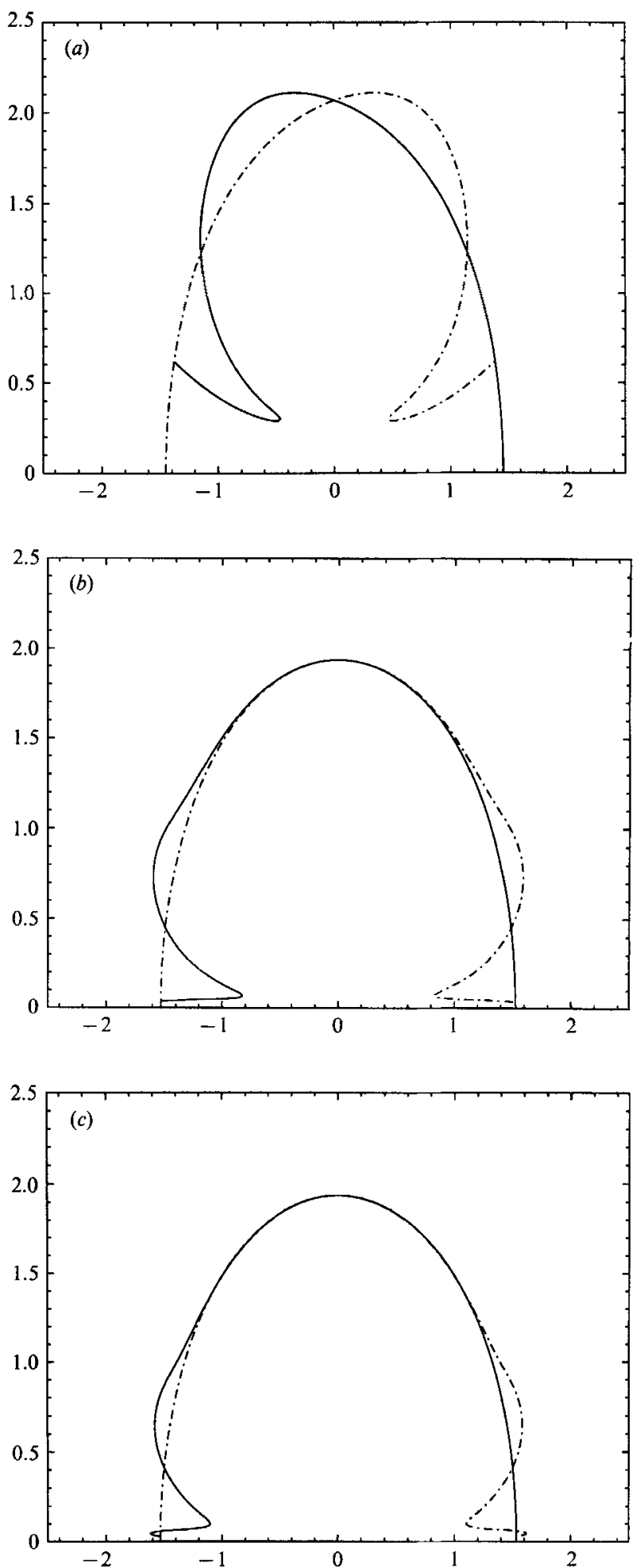

Figure $5(a-c)$. For caption see facing page. 

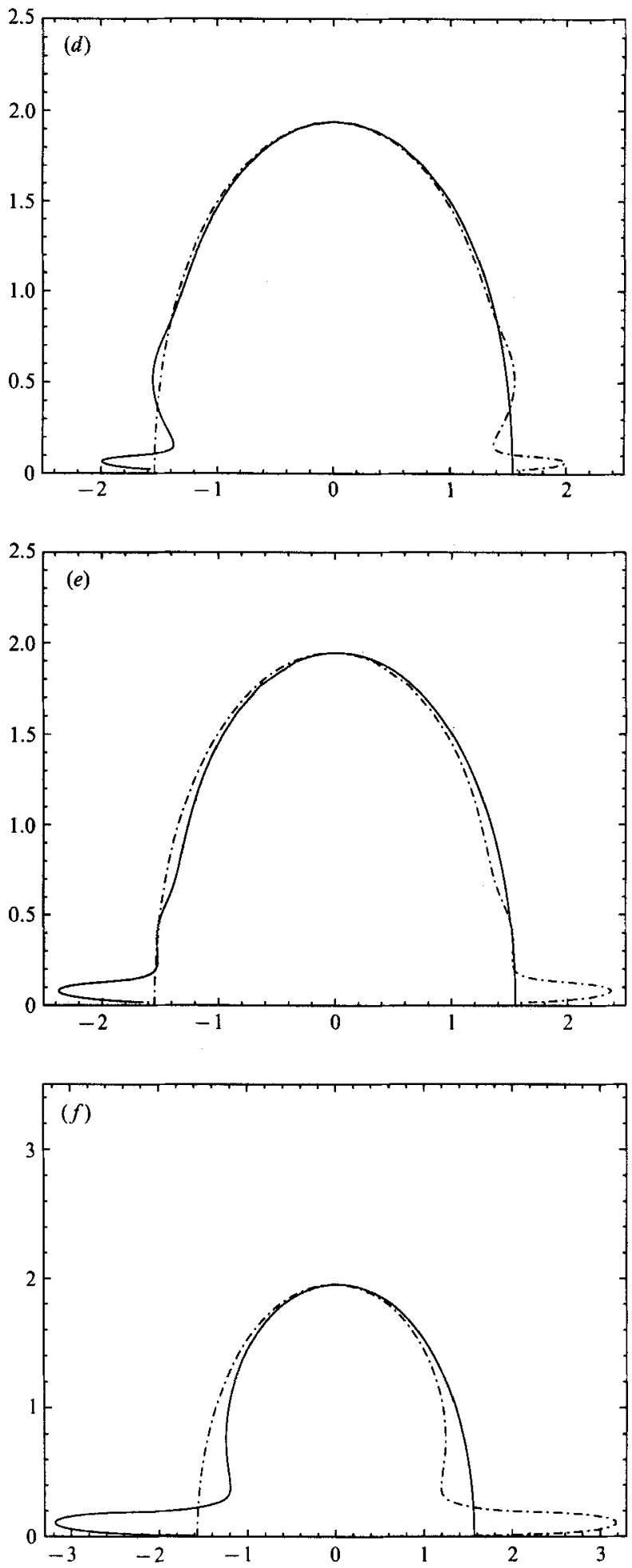

Figure 5. Numerical computations of the invariant manifolds for $\epsilon=0.1$ and various parameter values; $(a) \gamma=0.5,(b) 1.31,(c) 1.38,(d) 1.48,(e) 1.58,(f) 1.81$. - Unstable manifold; -....-, stable manifold. 


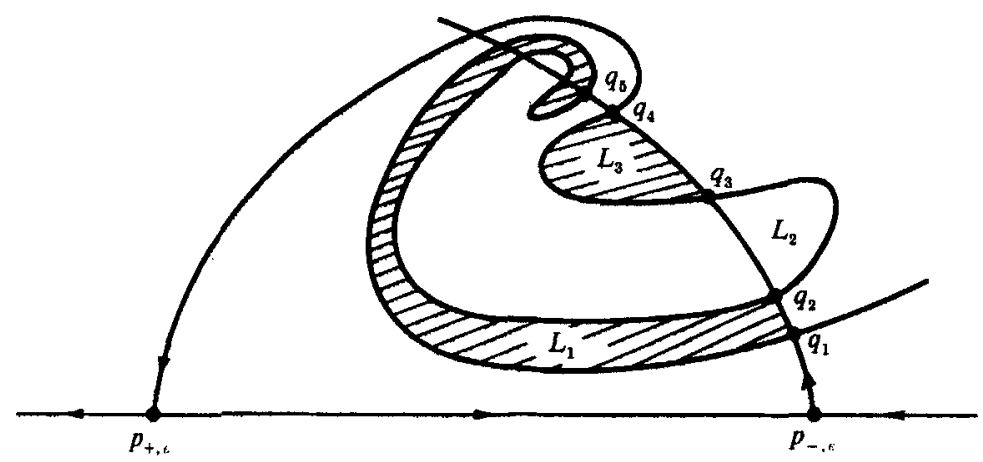

FigURe 6. $q_{1}, q_{2}, q_{3}, q_{4}$ are p.i.p.s, $q_{5}$ is not a p.i.p. $L_{1}, L_{2}, L_{3}$ are the lobes with $L_{1}<L_{2}<L_{3}$.

the geometrically complex structure associated with the tangling of the manifolds. This allows us to obtain a quantitative hold on the dynamics in the mixing region. In much of what follows there will be no restriction on the amplitude of $\epsilon$.

\subsection{Lobe motion}

We begin with two definitions.

Definition 5.1. Consider a point $q \in W_{+, \epsilon}^{\mathrm{s}} \cap W_{-, \epsilon}^{\mathrm{u}}$ and let $p_{+, \epsilon} q$ denote the segment of $W_{+, \epsilon}^{\mathrm{s}}$ from $p_{+, \epsilon}$ to $q$ and let $p_{-, \epsilon} q$ denote the segment of $W_{-, \epsilon}^{\mathrm{u}}$ from $p_{-, \epsilon}$ to $q$. Then $q$ is called a primary intersection point (p.i.p) if $p_{+, \epsilon} q$ and $p_{-, \epsilon} q$ intersect only in $q$, i.e. $p_{+, \varepsilon} q \cap p_{-, \epsilon} q=\{q\}$. See figure 6 .

Definition 5.2. Let $q_{1}$ and $q_{2}$ be two adjacent p.i.p.s., i.e. there are no p.i.p.s on the segment of $W_{+, \varepsilon}^{\mathrm{s}}$ and $W_{-, \varepsilon}^{\mathrm{u}}$ which connect $q_{1}$ and $q_{2}$. We refer to the region bounded by the segments of $W_{+, \epsilon}^{\mathrm{s}}$ and $W_{-, \epsilon}^{\mathrm{u}}$ which connect $q_{1}$ and $q_{2}$ as a lobe. See figure 6 .

The spatial structure of the manifolds provides a natural ordering by time which is useful when we discuss the flux as well as entrainment and detrainment. To describe this ordering we need the following definitions.

Definition 5.3. Let $q_{1}$ and $q_{2}$ be p.i.p.s. Then we say that $q_{1}<q_{2}$ if $q_{1}$ is closer than $q_{2}$ to $p_{-, e}$ in terms of distance along $W_{-, \epsilon}^{u}$.

Definition 5.4. Suppose that $L_{1}$ and $L_{2}$ are lobes. Then we say that $L_{1}<L_{2}$ if each of the p.i.p.s defining $L_{1}$ are less than or equal to each of the p.i.p.s defining $L_{2}$.

Now let $q$ be a particular p.i.p. and consider the region bounded by

$$
p_{+, \varepsilon} q \cup p_{-, \epsilon} q \cup\left[W_{+, \varepsilon}^{\mathrm{u}} \cap W_{-, \varepsilon}^{\mathrm{s}}\right] \text {. }
$$

We refer to this as region $A$. We will describe the motion of fluid across the boundary of $A$. There is no restriction on the choice of $q$. In figure 7 we choose $q$ rather arbitrarily for illustrative purposes. Later we choose $q$ so that $A$ corresponds as much as possible to the unperturbed core. See figure 10.

Definition 5.5. A lobe is called an exterior lobe if no part of its interior is contained in A. A lobe that is not an exterior lobe is called an interior lobe. 


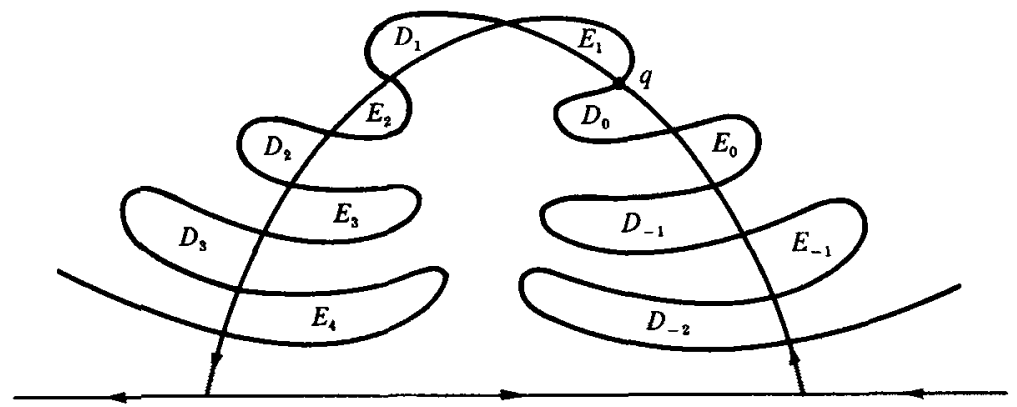

Figure 7. The $E_{i}$ and $D_{i}$.

Now consider figure 7 . The lobes $E_{i}$ are exterior lobes for $i \leqslant 0$ and interior lobes for $i>0$. Similarly, the lobes $D_{i}$ are exterior lobes for $i>0$ and interior lobes for $i \leqslant 0$. The following is our main result concerning the dynamics of the lobes.

Suppose that the lobes are defined so that for some $n \geqslant 1$ :

$$
T\left(E_{i}\right)=E_{i+n}, \quad T\left(D_{i}\right)=D_{i+n} .
$$

For small $\epsilon, n$ is just one-half the number of simple zeros of the Melnikov function in one cycle, $\tau=2 \pi \gamma$. (See Appendix D.) For arbitrary $\epsilon$ we construct $W_{+, \epsilon}^{\mathrm{s}}$ and $W_{-, \epsilon}^{\mathrm{u}}$ numerically and simply track the progression of a p.i.p. during one cycle to determine $n$.

The concise observation above belies its many underlying implications: (i) After one cycle of the time-periodic strain-rate field (i.e. one iterate of the Poincare map) $E_{0}, \ldots, E_{-n+1}$ enter region $A$. Similarly, after one cycle $D_{-1}, \ldots, D_{-n}$ leave region $A$. (ii) The lobes $E_{i}, D_{i}$ maintain their ordering throughout their evolution in time under the action of the Poincaré map. i.e.

$$
\begin{array}{cc}
E_{i}<E_{j} & \text { implies } T^{k}\left(E_{i}\right)<T^{k}\left(E_{j}\right), \\
D_{i}<D_{j} & \text { implies } T^{k}\left(D_{i}\right)<T^{k}\left(D_{j}\right), \\
E_{i}<D_{j} & \text { implies } T^{k}\left(E_{i}\right)<T^{k}\left(D_{j}\right)
\end{array}
$$

for all $k$. This is a consequence of the fact that the Poincaré map preserves orientation and therefore the relative ordering of points along $W_{-, \varepsilon}^{u}$ is preserved.

\subsection{Lobe area}

Knowledge of the total area of the $n$ lobes, $E_{0}, \ldots, E_{n+1}$, would tell us the amount of fluid entering $A$ per cycle. We show that the Melnikov function gives this information for small $\epsilon$.

Consider figure 8 and the lobe $L$ defined by the p.i.p.s $q_{1}$ and $q_{2}$. Let us denote the infinitesimal element of arclength along $W_{-, \epsilon}^{\mathrm{u}}$ by $\mathrm{d} s$ and let $l(s)$ denote the perpendicular distance between $W_{+, \epsilon}^{\mathrm{s}}$ and $W_{-, \epsilon}^{\mathrm{u}}$. Then the area of $L$, denoted $\mu(L)$, is given by

$$
\mu(L)=\int_{q_{1}}^{q_{2}} l(s) \mathrm{d} s .
$$

Now $W_{+, \epsilon}^{\mathrm{s}}$ and $W_{-, \varepsilon}^{\mathrm{u}}$ can be approximated uniformly on semi-infinite time intervals (see Guckenheimer \& Holmes 1983) and, because these manifolds move only an $O(\epsilon)$ 


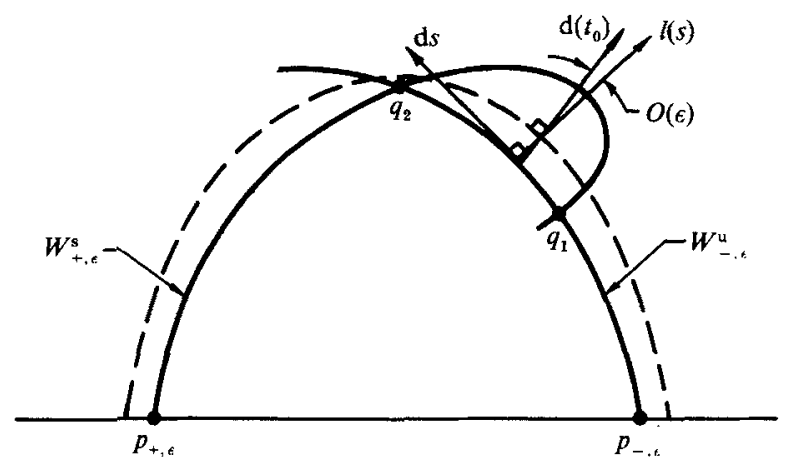

Figure 8. Geometry of the area of the lobes.

amount from the unperturbed manifolds on these time intervals, the angle between the line along which $d\left(t_{0}, \epsilon\right)$ is measured (see Appendix $C$ ) and the line along which $l(s)$ is measured is $O(\epsilon)$. Thus we can write

$$
\begin{aligned}
l(s) & =\left|d\left(t_{0}, \epsilon\right)\right|+O\left(\epsilon^{2}\right) \\
& =\frac{\epsilon\left|\boldsymbol{M}\left(t_{0}\right)\right|}{\left\|f\left(\boldsymbol{q}_{\mathrm{u}}\left(-t_{0}\right)\right)\right\|}+O\left(\epsilon^{2}\right)
\end{aligned}
$$

and

$$
\mathrm{d} s=\frac{\mathrm{d} s}{\mathrm{~d} t_{0}} \mathrm{~d} t_{0}=\left(\left\|f\left(\boldsymbol{q}_{\mathrm{u}}\left(-t_{0}\right)\right)\right\|+O(\epsilon)\right) \mathrm{d} t_{\mathbf{0}} .
$$

Substituting (5.2) and (5.3) into (5.1) gives

$$
\begin{aligned}
\mu(L) & =\int_{t_{01}}^{t_{02}}\left[\frac{\epsilon\left|M\left(t_{0}\right)\right|}{\left\|f\left(\boldsymbol{q}_{\mathrm{u}}\left(-t_{0}\right)\right)\right\|}+O\left(\epsilon^{2}\right)\right]\left(\left\|f\left(\boldsymbol{q}_{\mathrm{u}}\left(-t_{0}\right)\right)\right\|+O(\epsilon)\right) \mathrm{d} t_{0} \\
& =\epsilon \int_{t_{01}}^{t_{02}} M\left(t_{0}\right) \mid \mathrm{d} t_{0}+O\left(\epsilon^{2}\right),
\end{aligned}
$$

where $q_{\mathrm{u}}\left(-t_{01}\right)=q_{1}$ and $q_{\mathrm{u}}\left(-t_{02}\right)=q_{2}$. Thus we see that the integral of the Melnikov function between two adjacent p.i.p.s gives an $O(\epsilon)$ approximation to the area of the lobe defined by the p.i.p.s.

Several comments are now in order. (i) The validity of (5.4) relies heavily on the validity of the approximation of the perturbed manifolds via regular perturbation theory which is rigorous only on semi-infinite time intervals. Thus (5.4) is only valid for lobes defined by p.i.p.s that are outside of sufficiently small neighbourhoods of $p_{+, \epsilon}$ and $p_{-, \epsilon}$. However, in our case, the Poincaré map preserves area, so knowing the area of one lobe implies that we know the area of all the images of that lobe under the Poincaré map. (ii) For our problem, substituting (4.3) into (5.4) gives the following expression for the area of the $n$ lobes:

$$
\mu(L)=2 \epsilon|F(\gamma)|+O\left(\epsilon^{2}\right) .
$$

This gives us the explicit dependence of the area of the lobes on the parameter $\gamma . \mathrm{A}$ comparison between the numerical calculation of lobe area and the analytical result (5.5) gives good agreement as seen in figure 9 .

Now let us return to our specific problem. We choose the region $A$ to be defined by the p.i.p. that lies on the $y$-axis. As mentioned above, this is so that the resulting 


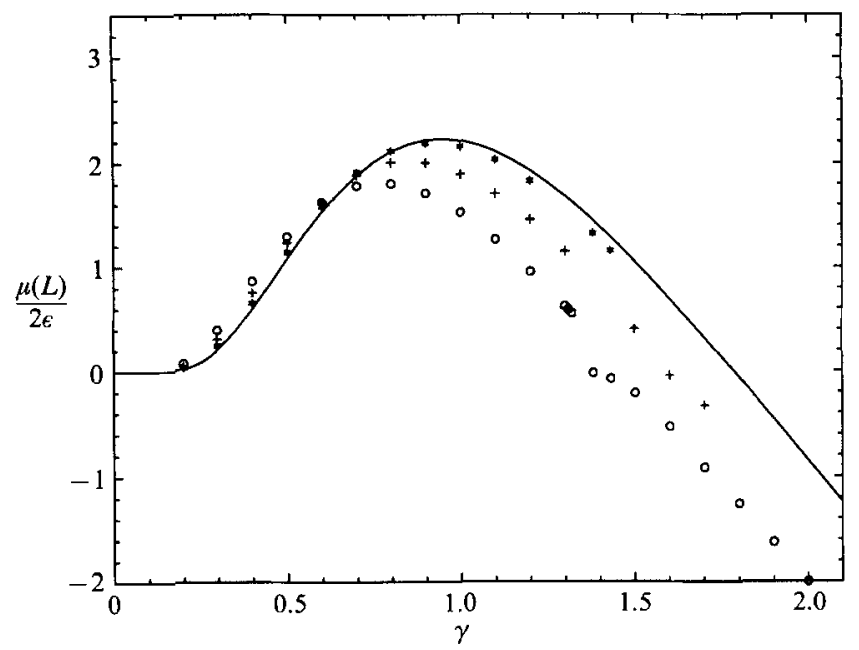

Figure 9. Comparison between theoretical and numerical calculations of lobe areas: - $-F(\gamma)$; $*, \epsilon=0.01 ;+, \epsilon=0.05 ; O, \epsilon=0.1$.

(a)

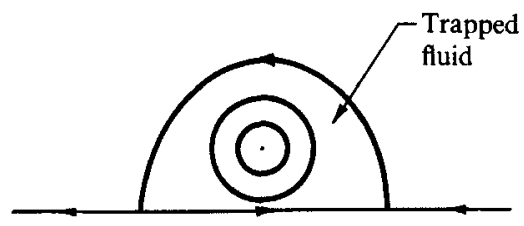

(b)

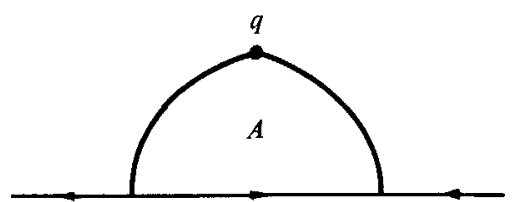

Figure 10. The geometry of region $A ;(a)$ unperturbed flow; $(b)$ perturbed flow.

shape of the region is very similar to the region of trapped fluid in the unperturbed velocity field, see figure 10 .

From (4.3), $M\left(t_{o}\right)=(F(\gamma) / \gamma) \sin t_{0} / \gamma$ and, therefore, for small $\epsilon$, one lobe enters and one lobe leaves the region $A$ during each cycle, with the area of each lobe equal to $2 \epsilon|F(\gamma)|+O\left(\epsilon^{2}\right)$. Notice from figure 9 that for $\gamma \approx 0.93, F(\gamma)$ obtains its maximum, thus the volume of fluid entrained per cycle is maximal at this $\gamma$. The volume entrained per unit time is maximum at $\gamma \approx 0.7$ when $F(\gamma) / \gamma$ is maximum. For $\gamma \approx$ 1.78 the Melnikov function vanishes identically. Hence the $O\left(\epsilon^{2}\right)$ terms in the formula for the distance between the manifolds become important. For example, for $\epsilon=0.1$, we see in figure $5(c)$ that the manifolds are nearly tangent for $\gamma=1.38$ rather than for 1.78. Numerically, we observe also that near the $\gamma$ for which the Melnikov function vanishes two lobes enter and leave the region $A$ per cycle. (See figure $5 c, d, e$.

Finally we note that one must, in general, be on the lookout for the creation of additional hyperbolic fixed points as the perturbation parameter is varied, as in the blinking vortex flow (Khakhar et al. 1986). In the present flow we expect one or more 
such points and their homoclinic tangles to be created within the core as $\epsilon$ increases from zero and, as $\epsilon$ increases further and the last KAM torus moves inward, some of these points and their tangles may reside in the mixing region. At this point one could refine the notion of transport by dividing $A$ into appropriate subregions and considering transport between the subregions, as discussed by Rom-Kedar \& Wiggins (1989). We do not consider such refinements in this paper and confine our discussion in the next section to particle transport in and out of region $A$.

\section{Particle transport}

The time spent in $A$ depends on the particle's initial conditions and we define this time as the residence time. A volume of fluid therefore has an associated residence time distribution.

The notion of a residence time distribution is an important concept in mixing systems. For example, if fluid is injected into a catalytic reactor, the amount of product will be primarily influenced by the time spent by the fluid in the reactor. Other processes such as chemical reaction and heat or mass transfer have similar dependencies on the residence time distribution. Danckwerts (1953) discussed the importance and the application of this notion for steady flows through vessels (such as a pipe or tank) and the work presented here is similar to his in spirit. Both works rely on the simple observation that in order to determine the residence time distribution of the fluid initially in the vessel or in $A$, one needs to know the future of entering fluid only. Though developed separately, the method deseribed here can be thought of as a discretization of Danckwert's work to maps, where again the advantage of working with the Poincaré map instead of the time-dependent flow is apparent.

We refer to the motion of fluid into $A$ as entrainment and the motion of fluid out of $A$ as detrainment. For our problem the Melnikov function has two simple zeros per period and therefore (at least for sufficiently small $\epsilon$ ) one lobe is entrained and one lobe is detrained per cycle. We denote these lobes by $E$ and $D$, respectively, see figure 11. This implies that the volume of fluid entrained into region $A$ during each cycle is the area of lobe $E$ or $\mu(\mathbf{E})$. Also, the amount detrained from region $A$ during each cycle is the area of lobe $D$ or $\mu(D)$ and by incompressibility it must be equal to $\mu(E)$ and, in general,

$$
\mu\left(T^{l} D\right)=\mu\left(T^{k} E\right) \quad \text { for all } l, k=0, \pm 1, \pm 2, \ldots
$$

In this section we discuss more detailed questions of particle transport which can be answered by applying the following rules:

R1. Fluid entering region $A$ on cycle $k$ must be in $E$ on cycle $k-1$.

R2. Fluid leaving region $A$ on cycle $k$ must be in $D$ on cycle $k-1$.

R3. $T^{l}(E)$ cannot intersect $T^{k}(E)$ and $T^{l}(D)$ cannot intersect $T^{k}(D)$ for any $k$, $l=0, \pm 1, \pm 2, \ldots$.

Regarding R3, we note that it is possible for $T^{l}(E)$ to intersect $T^{k}(D)$ for some integers $l$ and $k$.

The questions we wish to address are: (1) How long does it take fluid to escape $A$ given that it started in $A$ ? Remarkably, it will turn out that answering this question is equivalent to answering the following question: (2) How long does it take fluid to escape from $A$ given that it is in lobe $E$ initially?

More especially we wish to determine the residence time distributions for the two initial conditions mentioned above. The answer to question (1) may be obtained by 

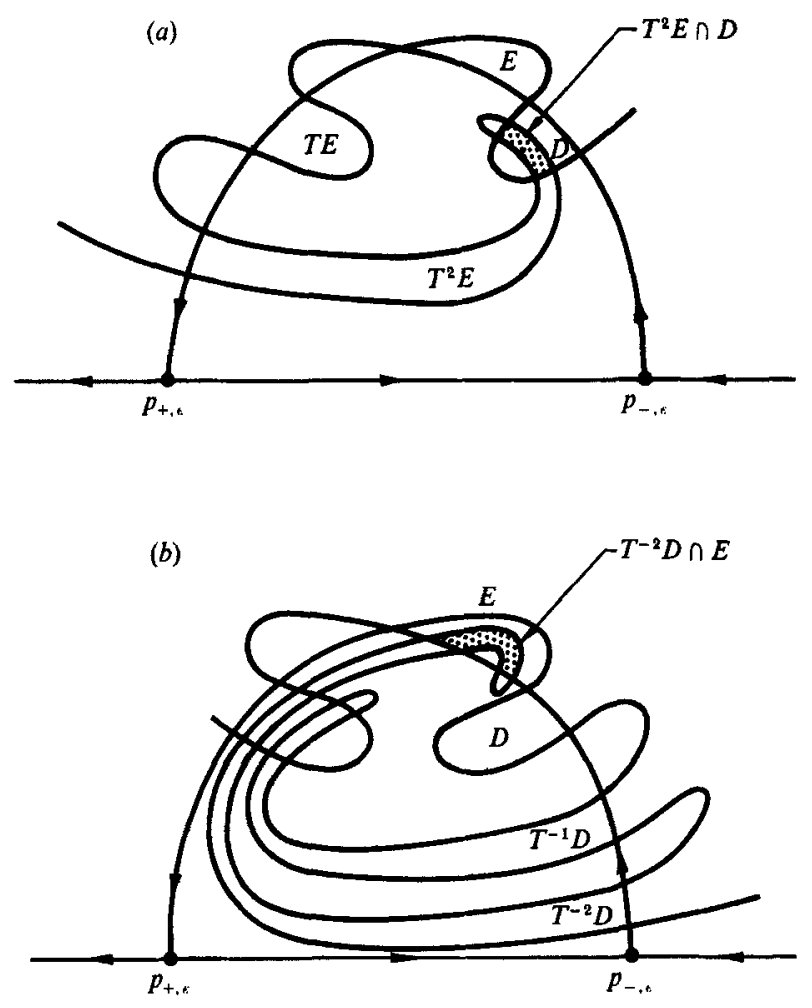

Figure 11. (a) $e_{3}=\mu\left(T^{2} E \cap D\right),(b) e_{3}=\mu\left(E \cap T^{-2} D\right)$.

brute force calculations, where a large number of initial conditions in $A$ are integrated and the number escaping each cycle are counted. The results of such calculations for two $\gamma$ values are presented in figure $20(a, b)$. The similarity to the manifold's shape as seen in figure 3 and demonstrated in figure $20(c)$ is not accidental, it is a manifestation of the lobe dynamics as described in \$5. Using the lobe dynamics enables us to reduce the problem to the computation of the residence time distribution for lobe $E$ only.

\subsection{Escape rates}

We consider first fluid that is in lobe $E$ initially (at cycle 0 ). As discussed previously, after one cycle the fluid in $E$ enters $A$. However, at some later time, say cycle $k-1$, a portion of the original fluid may be found in lobe $\mathrm{D}$ and therefore will escape $A$ on the next cycle. We define $e_{k}$ to be that portion, i.e.

$e_{k}=$ volume of fluid in lobe $E$ at cycle 0 that escapes $A$ on the $k$ th cycle.

Clearly,

$$
e_{k}=\mu\left(T^{k-1} E \cap D\right), \quad k=1,2, \ldots
$$

and

$$
e_{k}=0, k \leqslant 0 \text {. }
$$

Note that the corresponding distribution of escape times is given by $e_{k} / \mu(E)$. Using incompressibility $(6.2 a)$ can alternatively be written

$$
e_{k}=\mu\left(E \cap T^{-k+1} D\right), \quad k=1,2, \ldots
$$


Figure 11 illustrates the geometry associated with $(6.2 a)$ and (6.3). Note that replacing $E$ with $T^{-l} E$ in $(6.2 a)$ and (6.3) gives the volume of fluid in lobe $T^{-l} E$ at cycle 0 that escapes $A$ on the $k$ th cycle. This is clearly equal to $e_{k-1}$. In fact, the $e_{k}$ contain information concerning all possible intersections of any $E_{i}$ lobe with any $D_{j}$ because

$$
e_{k}=\mu\left(T^{k+m-1} E \cap T^{m} D\right), \quad m=0, \pm 1, \pm 2, \ldots .
$$

In figure 12 we illustrate the case $m=-k+1$ by displaying the sets $E \cap T^{-k+1} D$ for several values of $k$ and $\gamma=0.5,0.9$.

Now we consider the escape distribution for region $A$ and define escape volumes as follows:

$$
a_{k}=\text { volume of fluid in region } A \text { on cycle } 0 \text { that escapes on cycle } k \text {. }
$$

From previous discussions it follows that fluid leaving $A$ on the $k$ th cycle must be in the lobe $T^{-k+1} D$ at cycle 0 . However, not all of $T^{-k+1} D$ was in $A$ at cycle 0 since portions of $T^{-k+1} D$ may intersect $T^{-l} E, 0 \leqslant l \leqslant k$, and should not be counted. So it follows that

$$
a_{k}=\mu\left(T^{-k+1} D\right)-\sum_{l=0}^{k} \mu\left(T^{-k+1} D \cap T^{-l} E\right),
$$

where the sum in (6.4) represents the volume of $T^{-k+1} D$ that is also in some $T^{-l} E$ for $0 \leqslant l \leqslant k$. By incompressibility we have

$$
\mu\left(T^{-k+1} D\right)=\mu(D)=\mu(E)
$$

and from (6.3) we have

$$
e_{k-l}=\mu\left(T^{-k+1} D \cap T^{-l} E\right) .
$$

Using (6.6) and (6.5) allows us to simplify (6.4) as follows:

or

$$
\begin{gathered}
a_{k}=\mu(E)-\sum_{l=0}^{k} e_{k-l} \\
a_{k}=\mu(E)-\sum_{l=1}^{k} e_{l} .
\end{gathered}
$$

Thus, to compute $a_{k}$, we only need information concerning the dynamics of lobe $E$, namely the $e_{k}$. We find $e_{k}$ numerically by computing the escape cycle for each member of a regular array of grid points in lobe $E$. To verify the relation between the $e_{k}$ and the $a_{k}$ given by (6.8), we have also computed the $a_{k}$ for one particular choice of the parameters $\epsilon$ and $\gamma$ by a 'brute force' calculation using an array of grid poin's in region $A$. The results are shown in figure 13 and confirm (6.8).

We note that with little effort we can obtain other quantities which are of physical interest in terms of the $e_{k}$ such as

$$
r_{k}=\text { volume of fluid initially in } A \text { that remains in } A \text { after } k \text { cycles. }
$$

Clearly,

$$
\begin{gathered}
r_{k}=r_{k-1}-a_{k} \\
r_{k}=\mu(A)-\sum_{i=1}^{k} a_{i} .
\end{gathered}
$$

Using (6.8) we obtain

$$
r_{k}=\mu(A)-k \mu(E)+\sum_{i=1}^{k}(k-i+1) e_{i}
$$


(a)

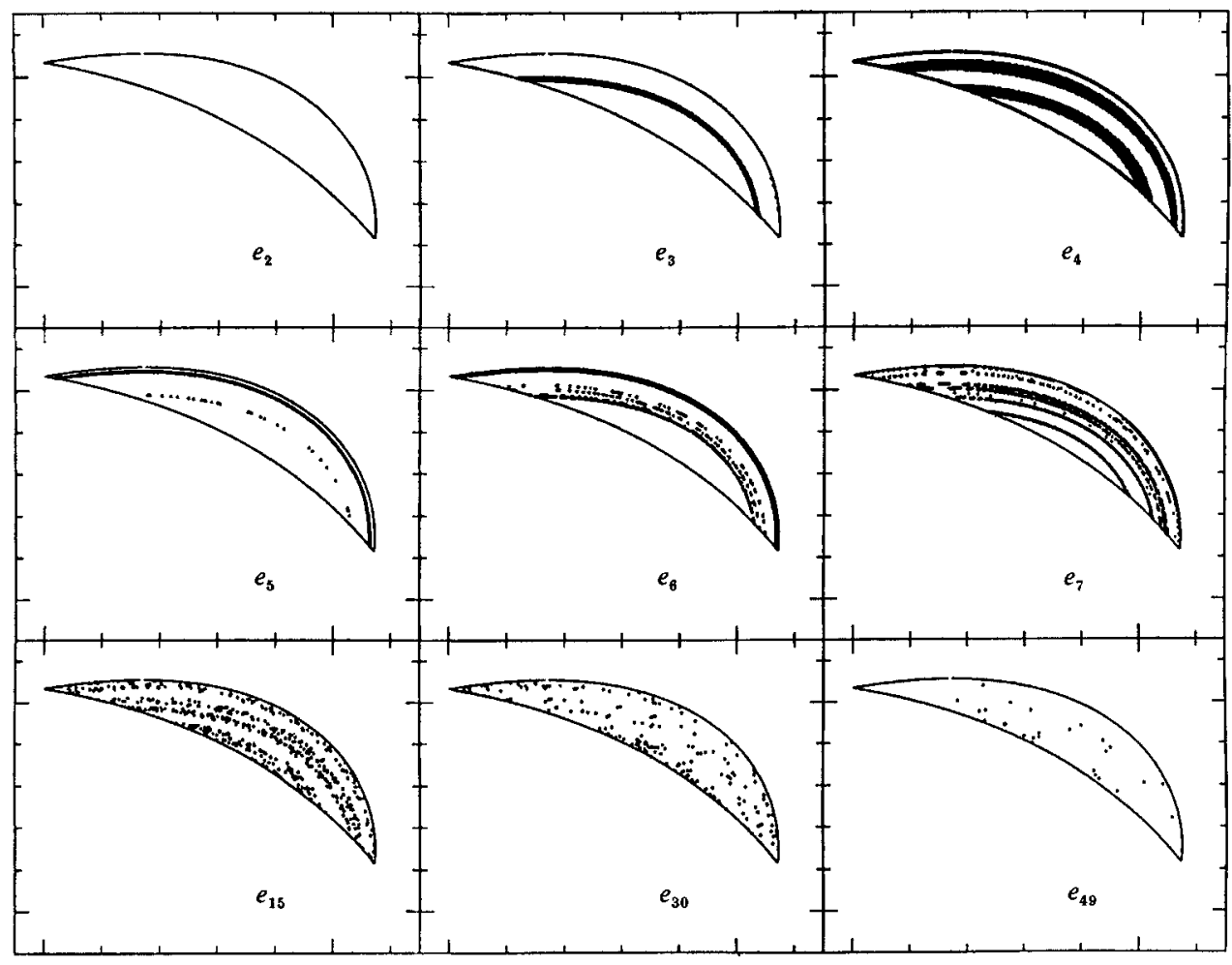

(b)

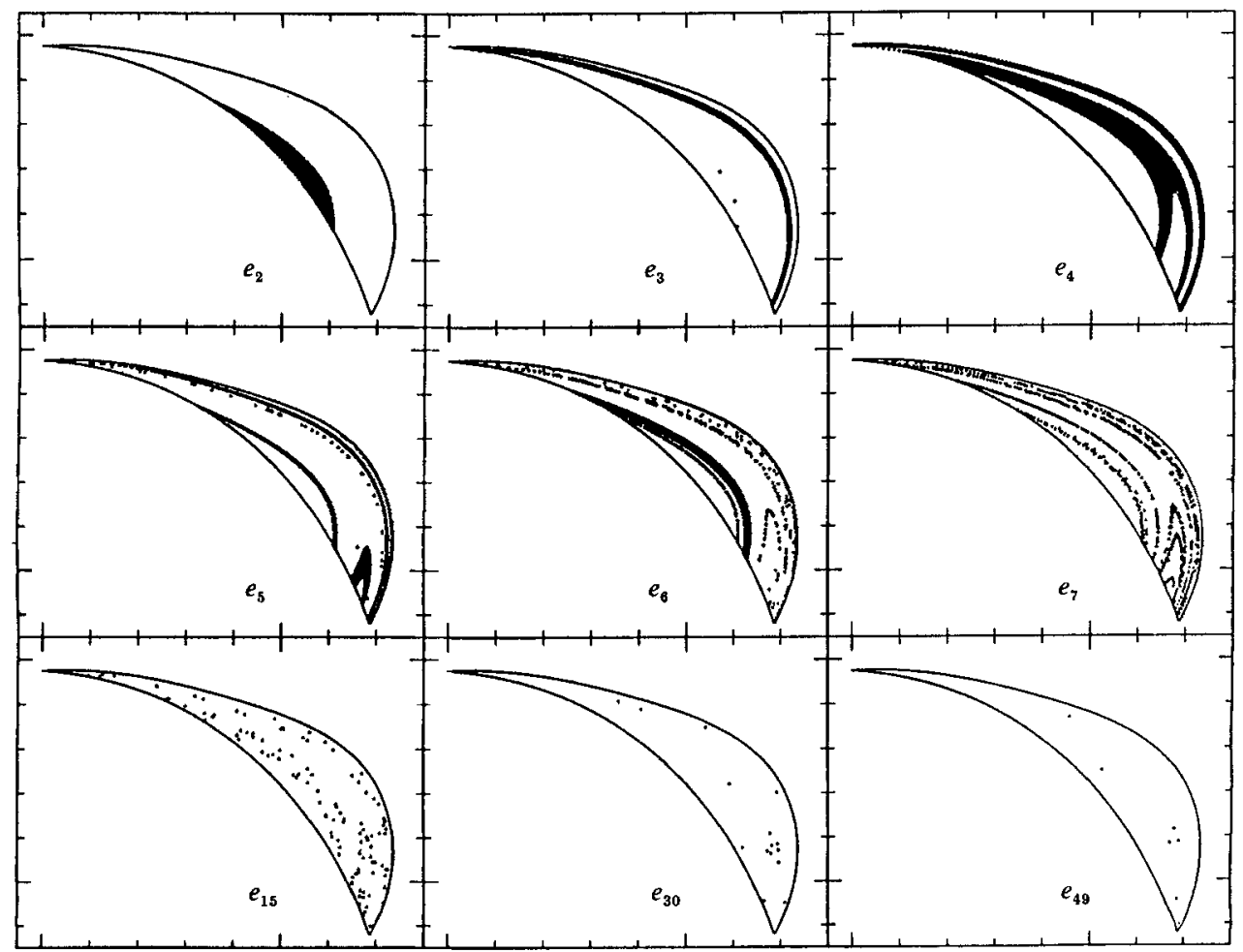

FIGURE 12. Geometry of $E \cap T^{-k+1} D$ for $\epsilon=0.1$ and various values of $k$. (a) $\gamma=0.5,(\beta) \gamma=0.9$. 


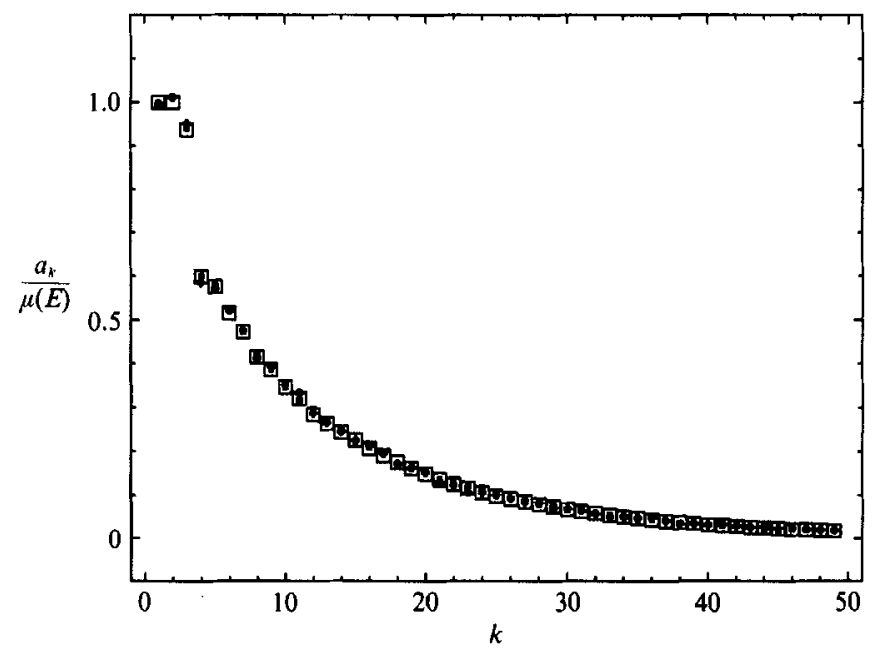

Figure 13. Comparison of the brute force calculation and the reduced calculation for $\gamma=0.5$; brute force calculation with mesh $=0.005 ; \square$, reduced calculation with mesh $=0.005$; \& , brute force calculation with mesh $=0.0075$.

Since $e_{k}$ and $r_{k}$ are finite and positive and $\sum_{i=1}^{k} e_{i} \leqslant \mu(E)$ for all $k$, we deduce, letting $k \rightarrow \infty$, that

$$
\begin{gathered}
\sum_{i=1}^{\infty} e_{i}=\mu(E), \\
\sum_{i=1}^{\infty}(i-1) e_{i}=\mu(A)-\mu(C),
\end{gathered}
$$

where $r_{\infty}=\mu(C) \leqslant \mu(A)$ and $\mu(C)$ is the volume of fluid initially in $A$ that never escapes, i.e. the volume of the core of $A$. The relation (6.12), stating that all particles that enter region $A$ will eventually escape $A$, is also evident from incompressibility. We note also the inversion formula

$$
e_{k}=r_{k}-2 r_{k-1}+r_{k-2} \text {. }
$$

\subsection{The mixing region}

We are now in a position to precisely define the mixing region. By definition, fluid particles not in either the free flow region or the core must have trajectories on the Poincare map that enter and leave the region $A$. Now in order to enter $A$ the fluid particle trajectory must be in $T^{-k} E, k=0,1,2, \ldots$ In order to leave $A$ the fluid particle trajectory must also be in $T^{-k} D, k=0,1,2, \ldots$ From (6.12) the lobe $E$ (and, hence, by invariance, all iterates of $E$ ) is completely filled with pieces of $T^{k} D$, for all $k$. Hence the mixing region is given by

$$
\bigcup_{k=-\infty}^{\infty} D_{k}
$$

The significance of this definition is that it allows us to characterize the region of extremely complex fluid motion in terms of the motion of a fluid line element of finite length (i.e. the boundary of the lobes $E$ and $D$ ). We note also that (6.15) gives us an additional characterization of the mixing region. Namely, fluid particle trajectories in the mixing region must make at least one revolution around a vortex. 
(a)

(b)
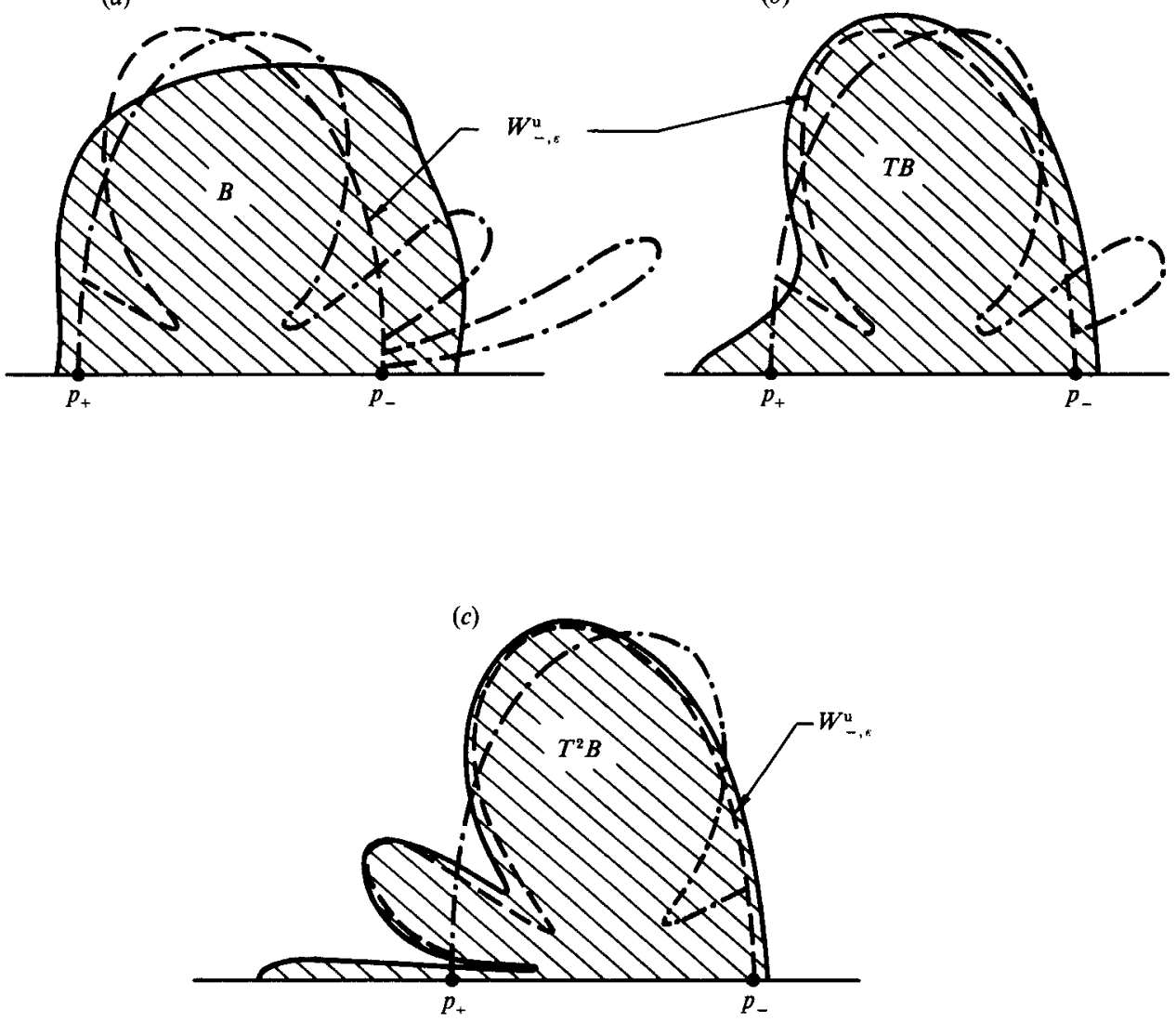

Figure 14. The motion of a general initial shape $B$; (a) $t=0,(b) t=2 \pi \gamma,(c) t=4 \pi \gamma$.

\subsection{The unstable manifold as the dominant organizing structure}

In general one would like to know the residence time distribution associated with any initial shape $B$ of finite area. This seems at the moment too difficult a question. Qualitatively one expects to have similar behaviour as obtained for $A$. Specifically, for any initial shape $B$ which can be regarded as distortion of $A$ (i.e. $B$ includes nontrivial parts of the mixing region, namely it is not contained in one $D$ lobe), the dominant structure which will be visualized and which will control the transport is

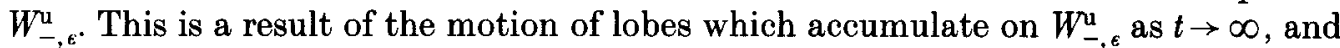
the assumption that $B$ has finite area. For example, consider the case in which $B$ overlaps $A$ on the upstream side. For large enough $k^{*}, T^{-k} E$ contains a very small portion of $B$ for all $k>k^{*}$, which implies that for $k>k^{*}, T^{k} B$ will have a very narrow layer upstream of $W_{-, \epsilon}^{\text {, }}$, see figure 14 . The above arguments apply to a broad class of flows having similar structure, namely hyperbolic stagnation points with cyclic motion near them, implying that the unstable manifold is the observed structure in many flow visualizations, depending on how the fluid is marked. In many ways the manifold acts as an attractor although it cannot be in the usual sense because of incompressibility.

A remarkable demonstration of the role of the unstable manifold as an organizing structure is obtained by comparing the computations of the unstable manifolds for 


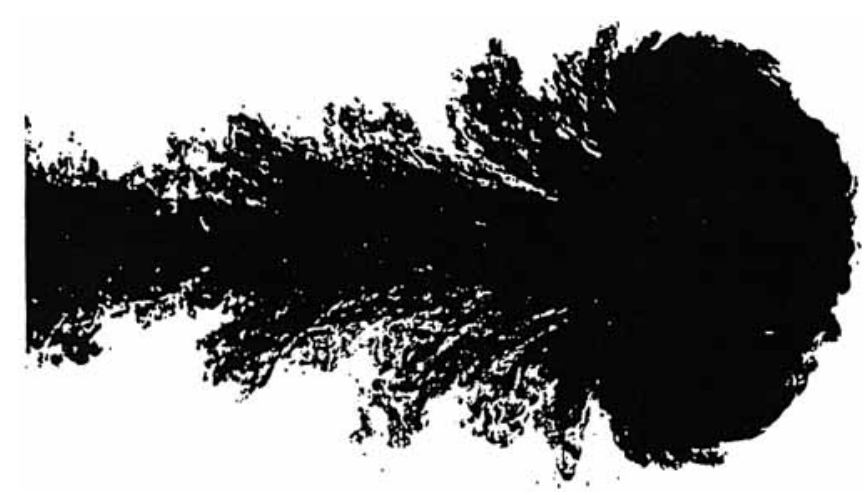

Figure 15. Flow visualization of a turbulent vortex ring.

(A. Glezer \& D. Coles 1987, private communication.)

particle motion in the presence of two idealized, leapfrogging vortex rings (Shariff et al. 1988, Shariff 1989) with flow visualization experiments of two vortex rings by Yamada \& Matsui (1978). These comparisons have been reproduced in Aref \& Kambe (1988, figure 8) and the reader is referred to them. In the idealized flow, the motion of the vortex rings and, therefore, the velocity field is periodic in time in a frame moving with the average speed of the rings. Ring motion is computed according to Dyson's (1893) model and consists of (1) an axial self-induced component that is proportional to $(1 / R(t) \log (R(t) / \delta(t))$, where $R$ is the ring radius and $\delta$ is the core radius $\left(R \delta^{2}=\right.$ const), plus $(2)$ the velocity contribution of the other ring. In the experiment, a smoke wire has stretched across the diameter of the pipe. Hence tracer is injected not only into the separating boundary layer which rolls-up to form the vortical cores, but also into the irrotational or weakly vortical fluid surrounding the cores. The smoke begins to reveal even the fine-scale features of the manifold.

As another example, consider transport in the vicinity of a single unsteady vortex ring. In the case of an ideal, steady axisymmetric ring, limiting streamsurfaces separate the fluid near the vortical core that moves with the ring from fluid in the free flow region and there is no exchange between the two regions. In most cases of interest, where the toroidal radius of the vortical core is not small compared to the ring radius, front and rear stagnation points will exist on the axis. For a turbulent vortex ring the velocity field is three-dimensional and unsteady corresponding, of course, to the three-dimensional motions of the vorticity field. Thus, threedimensional lobe-like structures continually pierce the ideal streamsurface from both sides and become severely distorted as they encounter the rear stagnation point of the ideal flow. These structures are revealed in the studies of A. Glezer \& D. Coles (1987, private communication) (see figure 15) where it is seen that marked fluid that is injected into the ring during formation is deposited into the wake in the form of three-dimensional lobes. Finally, in the case of ideal, unsteady axisymmetric vortex rings, Shariff (1989) has demonstrated computationally the presence of lobe structures in the Poincaré map for the flow in which the time-period perturbations are caused by the ellipticity of the vortex cores. 


\subsection{Numerical results and discussion}

We now present numerical results for the residence time distributions, $\left(e_{k}, a_{k}\right.$, and $r_{k}$ properly normalized) and discuss their dependence on $k$ and the parameter $\gamma$. In particular, some characteristics of the numerical results can be readily understood from the manifold structure.

The $e_{k}=\mu\left(E \cap T^{-k} D\right)$ can be computed in one of two ways.

1. Compute the boundaries of the lobes $E$ and $T^{-k} D$ and find the area of their intersection.

2. Track area elements of the interior of lobe $E$ to determine the area that escapes $A$ at each cycle,

Although the first method is theoretically more satisfying, there are two substantial difficulties: (1) the problem of the exponential stretch of the manifolds and thus of the lobe boundary and (2) the determination of the interior of a tangled boundary for large $k$. We therefore use the second method where the grid mesh on $E$ was chosen to be sufficiently small. In figure 12 , for large $k$, it appears as if the escape area is composed of a number of isolated area elements, but this is merely the result of using a finite number of non-deforming area elements. The actual areas in $E \cap T^{-k} D$ must be composed of a finite number of shapes that connect to each other or the boundary of $E$ since $T^{-k} D$ is simply connected. The appearance of isolated computational points for a relatively fine mesh $(\mathrm{d} x=\mathrm{d} y=0.005)$ shows that the widths of the interior regions of $E \cap T^{-k} D$ become extremely narrow and demonstrates the difficulties one would encounter when using a scheme that tracks the boundary of $E$ or $D$.

It is interesting to note how the quantities vary according to qualitative features of the manifolds. Figure 16 contains plots of $e_{k}$ for two $\gamma$ values. The rapid oscillations of $e_{k}$ with $k$ is typical for all values of $\gamma$ and is discussed below.

We include log-linear and log-log plots as an aid to identifying possible exponential or power-law behaviour but, because of the fluctuations of the $e_{k}$ for small and large $k$, we shall defer the discussion of these possibilities until the $a_{k}$ are presented. The small- $k$ fluctuations consist of two-cycle oscillations with even- $k$ maxima and odd- $k$ minima. We explain this phenomenon as follows. Note that the invariance of the manifolds gives $e_{2 k}=\mu\left(T^{2 k} E \cap D\right)=\mu\left(T^{k} E \cap T^{-k} D\right)$ and $e_{2 k-1}=\mu\left(T^{k-1} E \cap T^{-k} D\right)$. Now in the symmetric Poincaré map $T^{k} E$ and $T^{-k} D$ are mirror images of each other. Since near the $x$-axis both lobes are flat, we shall obtain, in general, a larger volume of intersection than is obtained in a 'transversal' intersection which occurs in the asymmetric intersection of $T^{k-1} E \cap T^{-k} D$. However, secondary intersections far from the neighbourhood of the stagnation point will relax this difference as $k$ increases. Thus the two-cycle oscillation of the $e_{k}$ decays. For larger $k(k \widetilde{>} 20)$ the fluctuations observed in figure 16 are due to the statistics of the computation. We have verified that a finer mesh will decrease these fluctuations.

The $a_{k}$ are shown in figure 17. Note that for small $k$ the $a_{k}$, as a function of $\gamma$, increase with $\gamma$ up to $\gamma \approx 0.8$ then decrease until $\gamma \approx 1.3$, then increase again. This behaviour is directly related to the entrainment rate or lobe area given by $2 \epsilon\left|F^{\prime}(\gamma)\right|+O\left(\epsilon^{2}\right)$ (see figure 9). Using figure $17(d-g)$ we can make tentative conclusions regarding the asymptotic behaviour of the $a_{k}$ for large $k$ for $\gamma=0.5$ and 0.9 . Figure $17(d)$ strongly indicates exponential behaviour for $\gamma=0.5$ or

$$
a_{k} \sim \beta\left(r_{k}-r_{\infty}\right) \text { as } k \rightarrow \infty,
$$

i.e. a constant probability of escape from $A-C$ for large $k$. For $\gamma<0.5$, similar 

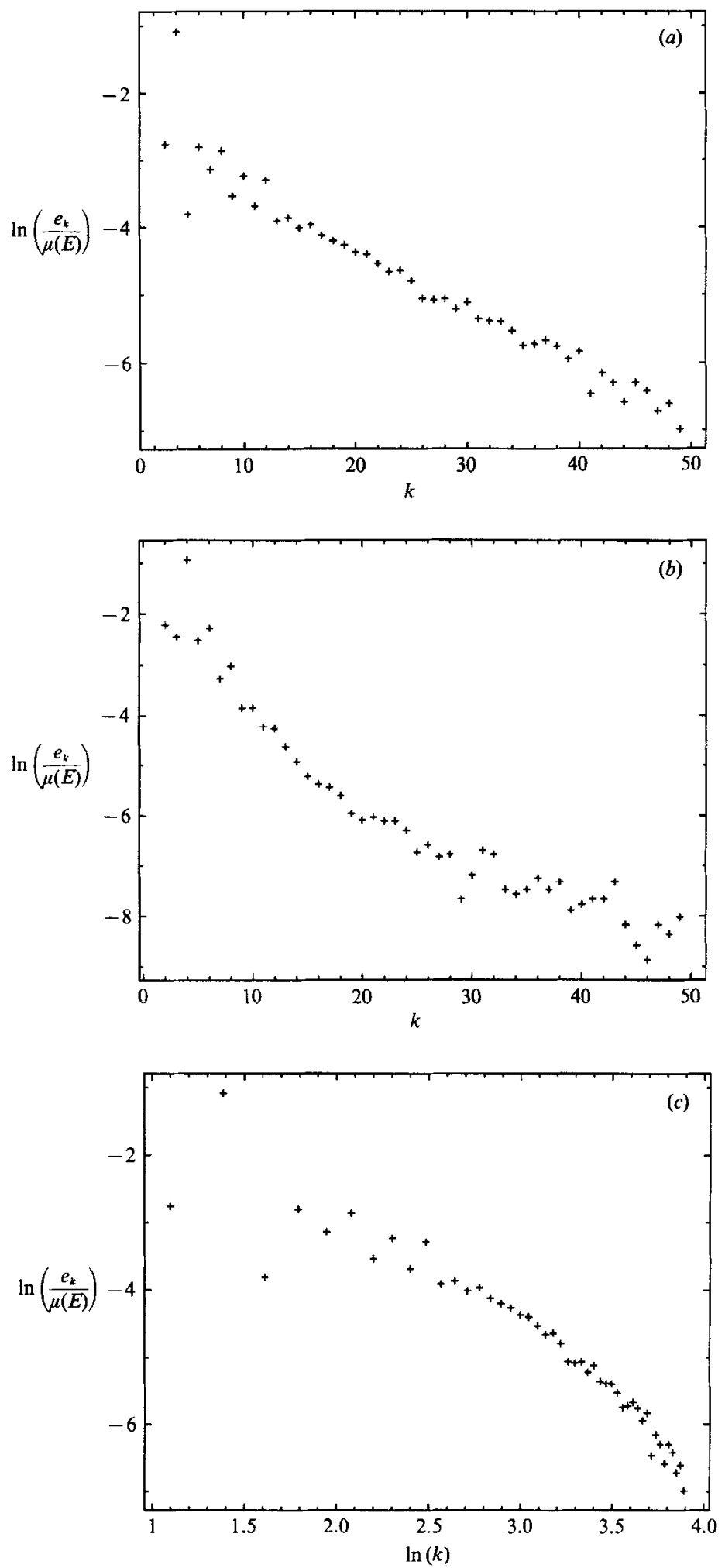

Figure $16(a-c)$. For caption see facing page. 


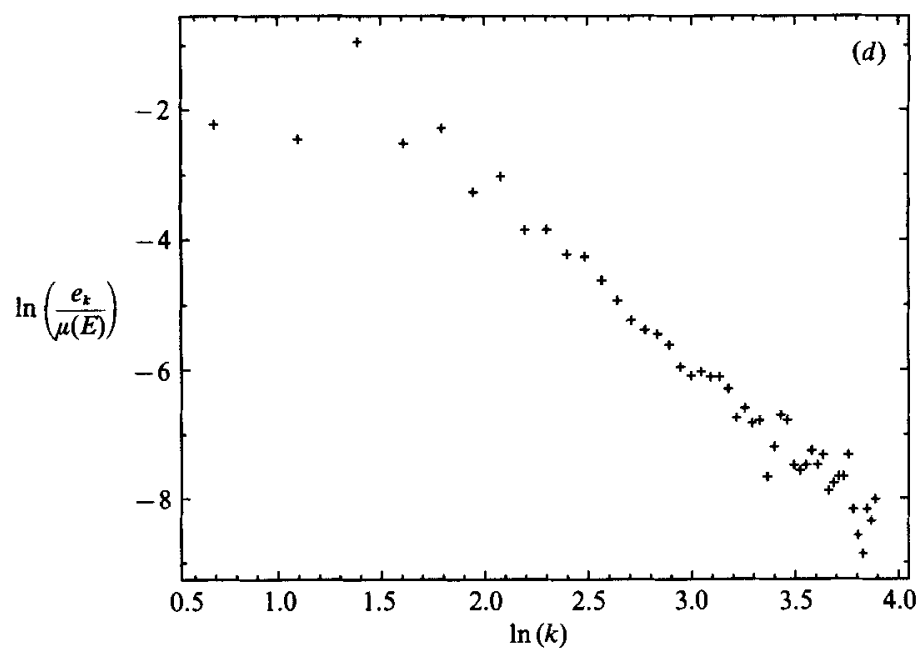

FIGURE 16. The $e_{k}:(a) \gamma=0.5$, log-linear plot; $(b) \gamma=0.9$, log-linear plot; (c) $\gamma=0.5, \log$-log plot; $(d) \gamma=0.9$, log-log plot.
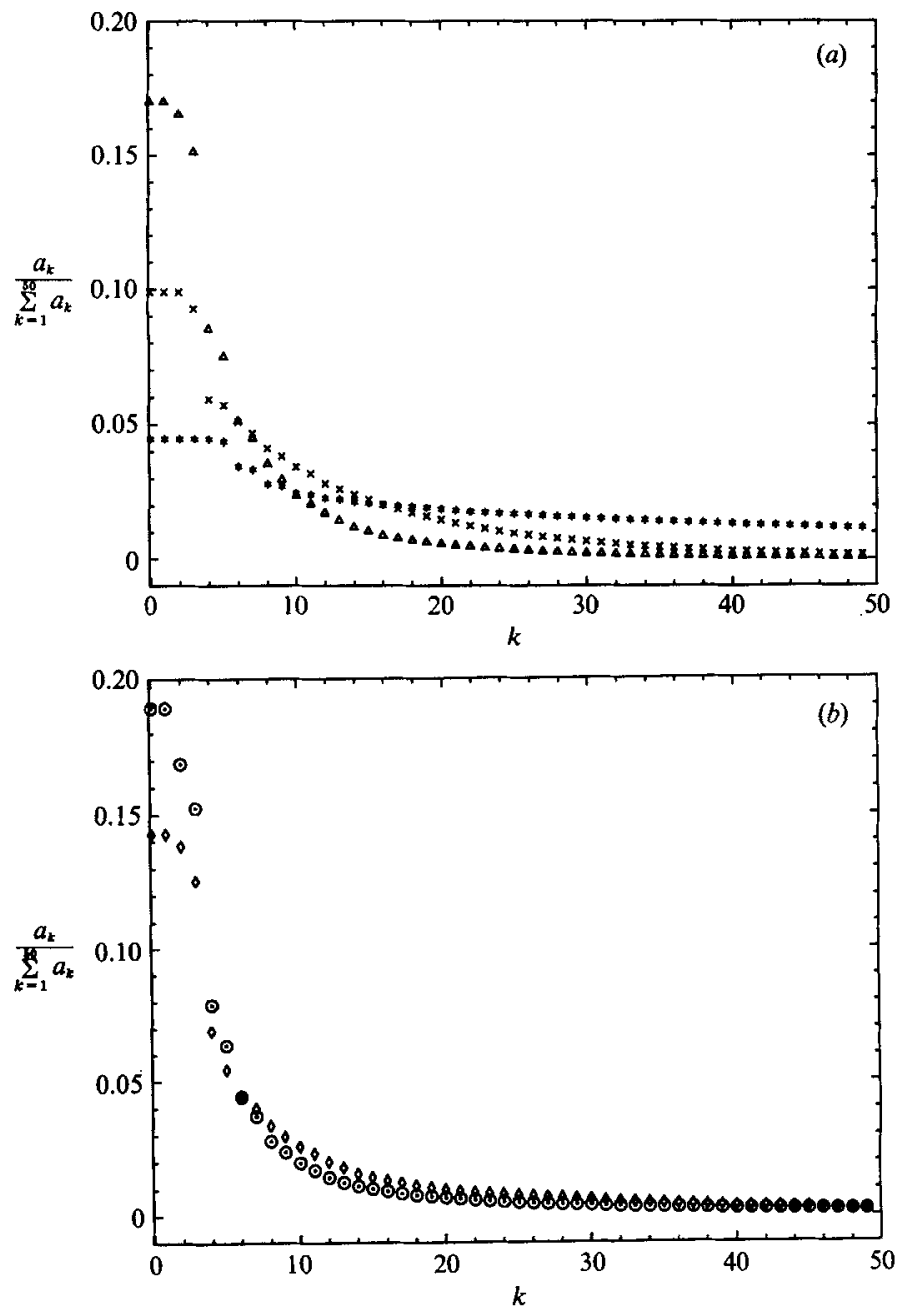

Figure $17(a, b)$. For caption see p. 375. 

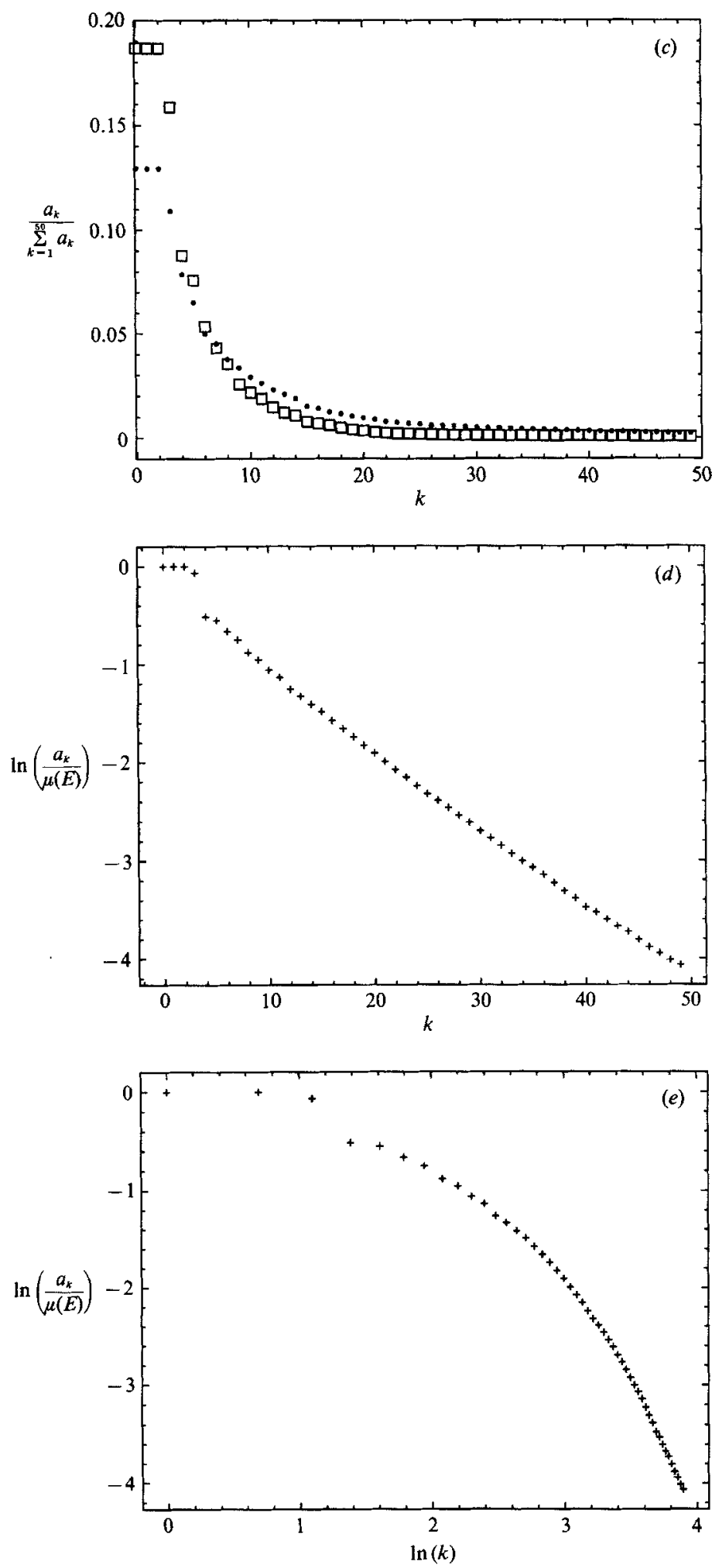

Frgure $17(c-e)$. For caption see facing page. 

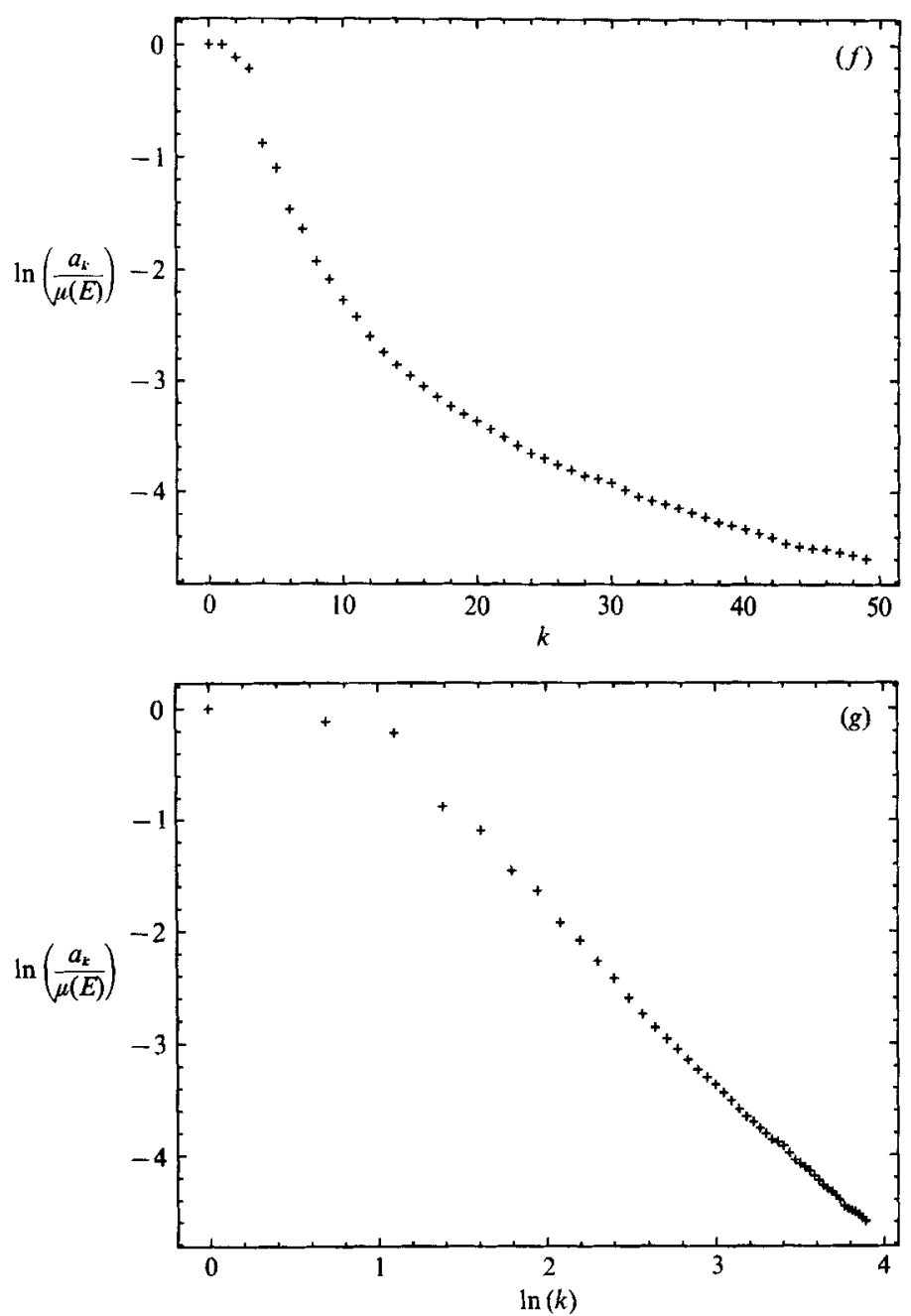

FIGURE 17. The $\left.a_{k} ;(a) \gamma=0.3\left(^{*}\right), 0.5(x), 0.7(\triangle) ;(b) \gamma=0.9(\odot), 1.1 \diamond\right) ;(c) \gamma=1.7(\odot), 1.9$ $(\square) ;(d) \gamma=0.5$, log-linear plot; $(e) \gamma=0.5, \log$-log plot; $(f) \gamma=0.9$, log-linear plot; $(g) \gamma=0.9$, $\log -\log$ plot.

exponential behaviour is indicated. For $\gamma>0.5$ there are no strong indications of either exponential or power-law behaviour with the possible exception of $\gamma=0.9$. For $\gamma=0.9$, figure $17(g)$ suggests a power-law behaviour for $a_{k}$ or

$$
a_{k} \sim \frac{\lambda}{k}\left(r_{k}-r_{\infty}\right)
$$

i.e. a decreasing probability of escape as $k \rightarrow \infty$.

Fitting an exponential for the $a_{k}$ by using a least-square method for the log-linear plots, we can compute $r_{\infty}$ using $(6.10)$ :

$$
r_{\infty}=r_{k_{0}-1}-\sum_{i=k_{0}}^{\infty} a_{i}=r_{k_{0}-1}-\frac{c \mathrm{e}^{-\alpha k_{0}}}{1-\mathrm{e}^{-\alpha}},
$$

where $c$ and $\alpha$ are related to the best linear fit coefficients. The results are presented 


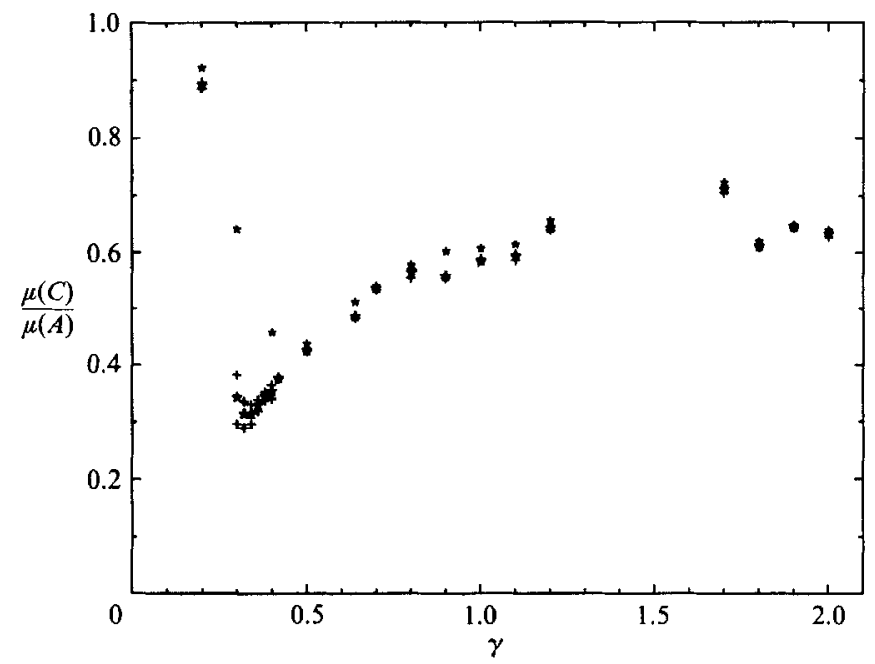

Figure 18. The core area:,$r_{50} / \mu(A)$; $r_{\infty} / \mu(A) ;+$, upper and lower bounds on $r_{\infty} / \mu(A)$.

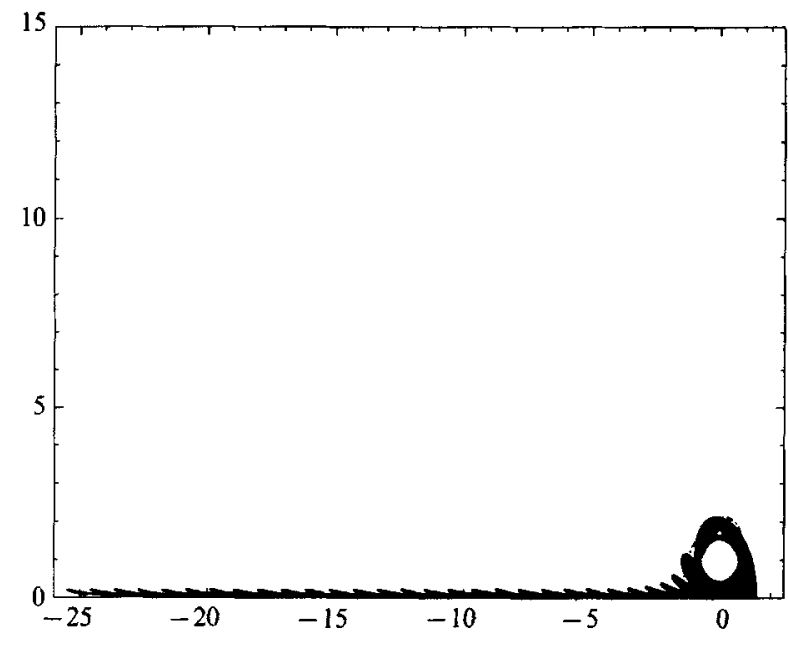

Figure 19. The unstable manifold for $\gamma=0.3$.

in figure 18 where $r_{50}, r_{\infty}$, and the bounds on $r_{\infty}$ computed via the least-square method are presented. The exponents we get are relatively small and therefore the linear fit to the log-linear plots, as well as the results for $r_{\infty}$, should be taken with caution.

The core $C$ is not necessarily composed of only one region, in fact, we find that the core splits into at least two separate domains for $\gamma=1.38$ for example. This is indicated from the photographs of the escape map (figure 20a,b, Plate 1), in which the red regions can be approximately identified with the core.

The appearance of a different number of regions with bounded motion for different values is the result of the distinctive resonances associated with each $\gamma$ value. Recall from $\$ 4$ that the streamline associated with a $p / q$ resonance is determined via the relation $T(I) / 2 \pi \gamma=p / q$. Therefore, as $\gamma$ increases, the streamline corresponding to the above relation has larger period and hence must be closer to the mixing region. Therefore, assuming that the largest KAM torus position is approximately 
(a)

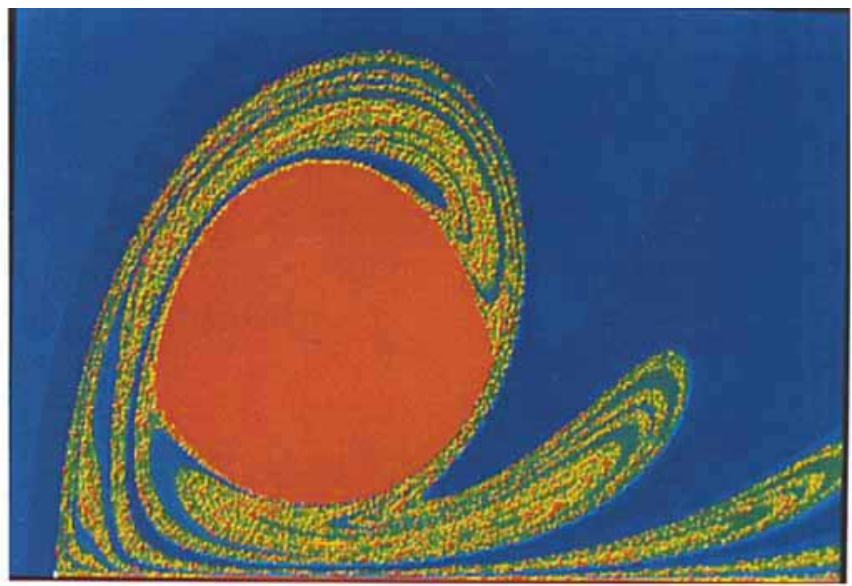

$$
\begin{aligned}
& \varepsilon=0.1 \\
& \gamma=0.5
\end{aligned}
$$

(b)

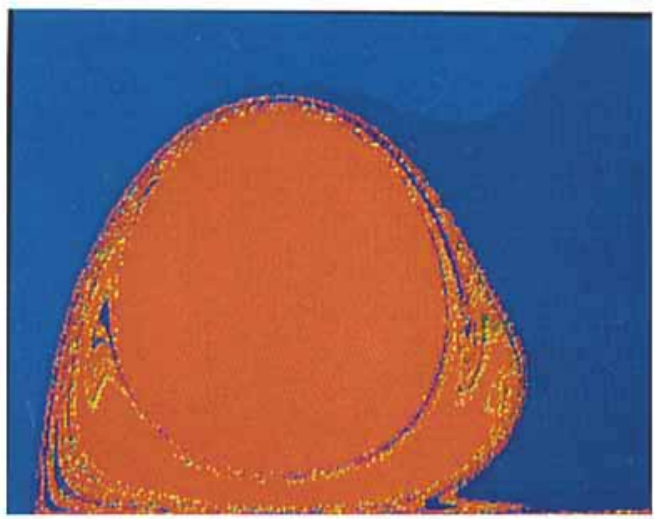

$$
\varepsilon=0.1
$$$$
\gamma=1.38
$$

(c)

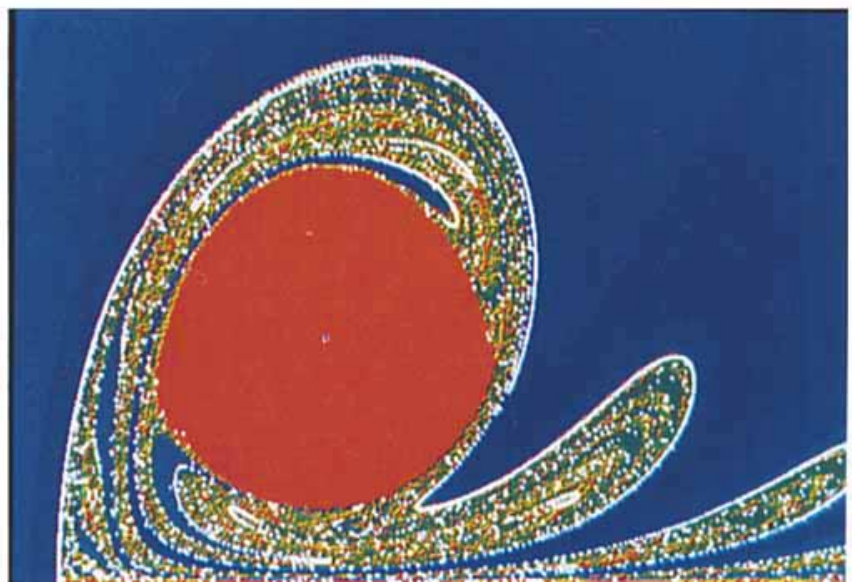

$$
\varepsilon=0.1
$$$$
\gamma=0.5
$$

FIGURE 20. The escape map for $(a) \gamma=0.5$, (b) $\gamma=1.38$, (c) $\gamma=0.5$ and $\varepsilon=0.1$ with the unstable manifold plotted in white. The initial conditions are coloured according to their residence time: the colour is graded from blue via green to red with residence time from 0 to more than 50 cycles. 
independent of $\gamma$, we expect that as $\gamma$ increases more resonance bands are present outside the largest KAM torus and with further increase in $\gamma$, they approach the manifolds and become unobservable. The scenario which is shown in figures 19 and 20 (Plate 1) fits the above description as follows:

For $\gamma=0.3$ the $p=4, q=1$ resonance band outside the largest KAM torus is manifested as four white spots outside the large white region - the main core. (In figure $20(c)$ the unstable manifold is plotted, and the white regions are the regions which the manifold cannot penetrate.) In figure $20(a)$, where the escape map for $\gamma=0.5$ is plotted we observe only one core region - the $q=1, p=4$ resonance band disappeared in the vicinity of the manifolds and no other resonance bands appear. As $\gamma$ increases to $\gamma=1.38$ the $p=1, q=1$ resonance band appears outside the largest $\mathrm{KAM}$ torus and two core regions are revealed in figure $20(b)$.

\section{Chaos}

We have seen that transport between the core and the free flow region can be understood by studying the interaction between the stable and unstable manifolds of $p_{+, \epsilon}$ and $\mathrm{p}_{-, \epsilon}$, respectively. Now we want to show that this interaction gives rise to another important dynamical effect, namely chaotic fluid particle motion.

Roughly speaking, chaotic fluid particle motion may result when structures in the flow conspire to strongly stretch, contract and fold a region of fluid. In our flow the tangling of the manifolds provides the folding mechanism and the fixed points $p_{+, \epsilon}$ and $p_{-, \epsilon}$ provide the stretching and contraction mechanism. The notion of chaos is made unambiguous when we show that this scenario enables us to prove that the Poincaré map possesses Smale horseshoes using the Smale-Birkhoff homoclinic theorem, see Guckenheimer \& Holmes (1983) or Wiggins (1988). Consider figure 21 and the 'rectangular' region of fluid denoted $R$. Following the evolution of this region under iteration by the Poincaré map $T$ we see that it is folded, stretched, and contracted and eventually mapped back over itself in the shape of a horseshoe.

We leave out the details but using techniques which can be found in Moser (1973) or Wiggins (1988) one can show that ' $R$ ' contains an invariant cantor set $A$ such that $\left.T^{n}\right|_{\Lambda}$, for some $n \geqslant 1$, has (i) a countable infinity of unstable periodic fluid particle motions of all possible periods; (ii) an uncountable infinity of unstable non-periodic fluid particle motions; (iii) a fluid particle whose orbit under $T^{n}$ eventually approaches every other point in $\Lambda$ arbitrarily closely. $A$ is called a chaotic invariant set for $T^{n}$. We remark that in Moser (1973) and Wiggins (1988) it is shown that by a continuous change of coordinates, $\left.T^{n}\right|_{A}$ can be transformed into a Bernoulli process hence making precise the notion of deterministic chaos. The construction shown in figure 21 could be repeated near any transverse heteroclinic point, hence Smale horseshoes and their associated chaotic dynamics exist throughout that part of the mixing region in $A$.

So the existence of transverse heteroclinic orbits in a heteroclinic cycle give rise to Smale horseshoes and are therefore the underlying mechanism for chaos. The Melnikov function allows us to determine if transverse heteroclinic orbits are present in the flow and hence give a specific criterion for the presence of Smale horseshoes in terms of the system parameters.

It should be apparent that the presence of horseshoes in a fluid flow may have a significant effect on neighbouring fluid particle motions. However, it is difficult to quantify this effect. Two things can be said. First, the unperturbed velocity field is 
378

V. Rom-Kedar, A. Leonard and S. Wiggins
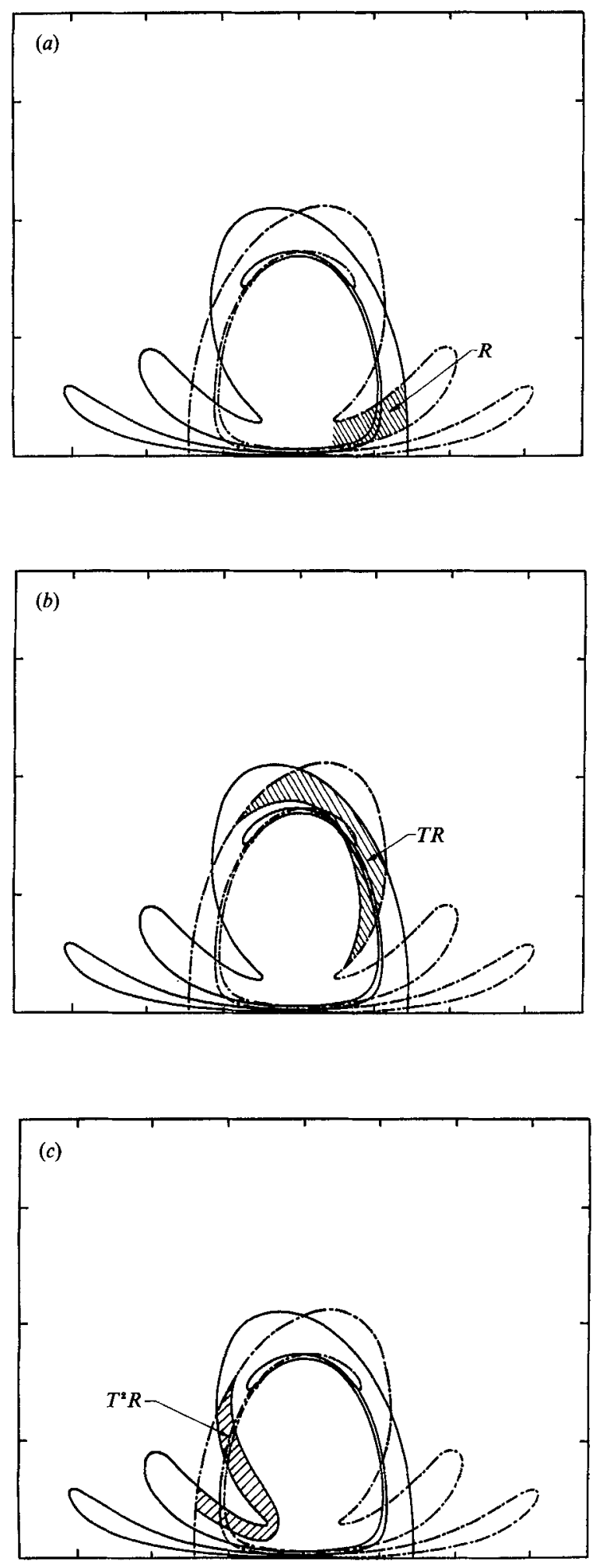

Figure $21(a-c)$. For caption see facing page. 


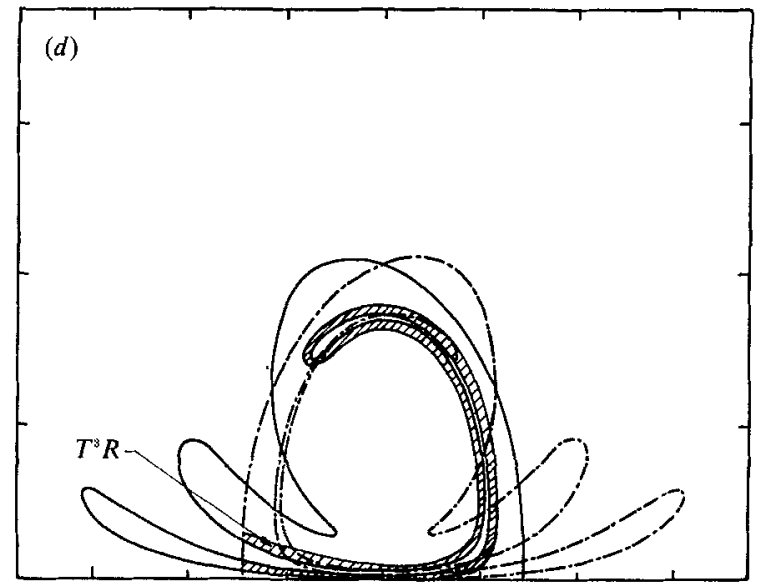

(e)

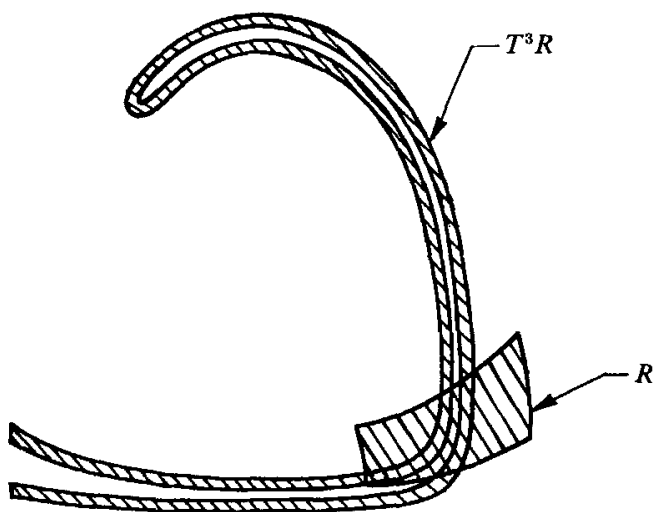

Figure 21. The geometry of the horseshoe map.

integrable; therefore typical fluid particles may separate at a linear rate at best. However, in the perturbed velocity field, nearby fluid particles may separate at an exponential rate and moreover the presence of horseshoes may cause fluid particle motions in the mixing region to become rapidly uncorrelated. Intuitively, one would believe that horseshoes are desirable in order to enhance mixing. We discuss these issues in the next section.

Secondly, in order to quantify the mixing of fluid between the core and the free flow region one must understand the dynamics of the interface, i.e. the stable manifold of $p_{+, \epsilon}$ and the unstable manifold of $p_{-, \epsilon}$. This is a topic which we are currently investigating in more detail. However, from our previous description a significant observation can be made. That is, in the unperturbed velocity field the interface separating the core and the free flow region has finite length but in the perturbed velocity field this interface has infinite length.

\section{Stretching and elongation of material elements}

In this section we investigate the rate of stretching of material elements in the oscillating vortex pair (OVP) flow and its relation to the time spent in the mixing region. The classical measure for quantifying the local stretching of material lines on 
the average is the Liapunov exponent, see for example, Khakhar et al. (1986). The motivation for computing the Liapunov exponent is that it quantifies the chaos in some systems; when a positive exponent exists, nearby trajectories diverge from each other exponentially. This notion is particularly useful when dealing with dissipative systems with attractors, since then all initial conditions will eventually diverge as much as trajectories on the attractor diverge. In the OVP flow we have proven the existence of chaotic orbits and we therefore have positive Liapunov exponents for those orbits. However, this set of chaotic orbits is of measure zero. Nevertheless, these orbits are responsible for the expansion and contraction in the mixing region: material elements passing through this region will experience the exponential stretching and contraction of the chaotic orbits. Most material points remain in this chaotic zone for only a finite time, which implies that their exponents vanish. Hence, in our application, the Liapunov exponent is not a useful measure of the stretching of material elements. To quantify this phenomenon we consider the total stretch or elongation of a material element due to its motion through the chaotic region. As discussed in $\$ 4$, we concentrate on analysing the behaviour in the mixing region.

We start by defining the stretching rate and its relation to the Liapunov exponent following the formulation of Khakhar et al. (1986). We write (2.3) together with (2.5) in the form

$$
\dot{\boldsymbol{p}}=F(\boldsymbol{p}, t),
$$

where $\boldsymbol{p}=(x, y)$. The linearized equation about an arbitrary solution of (8.1) is given by

$$
\dot{\boldsymbol{m}}=\boldsymbol{D}_{F}(\boldsymbol{p}(t), t) \boldsymbol{m},
$$

where $D_{F}$ is the matrix of partial derivatives of $F$.

We define the stretch of an infinitesimal material line $\mathrm{d} x$ emanating from $p$ with orientation $\boldsymbol{m}$ at $t=0$ as $\lambda(\boldsymbol{p}, \boldsymbol{m}, t)$. It is clearly given by

$$
\lambda(\boldsymbol{p}, \boldsymbol{m}, t)=\frac{|\boldsymbol{m}(t)|}{|\boldsymbol{m}|},
$$

where $\boldsymbol{m}(t)$ is a solution of $(8.2), \boldsymbol{m} \neq 0$. The Liapunov exponent of the orbit $p$ is defined as

$$
\sigma(\boldsymbol{p}, \boldsymbol{m})=\lim _{\boldsymbol{t} \rightarrow \infty} \frac{1}{t} \ln \lambda(\boldsymbol{p}, \boldsymbol{m}, t) .
$$

We now concentrate on the region of our interest, the mixing region. By $(6.15)$ this region is composed of $\bigcup_{k--\infty}^{\infty} D_{k}$; therefore we need to investigate the orbits in the $D_{k}$ lobes only. While $p$ is in the chaotic region, we expect, by definition, that the instantaneous stretching rate, $\dot{\lambda} / \lambda$, will have a positive average. After $\boldsymbol{p}$ escapes however, we expect stretching to decrease substantially. In fact we have been able to show (Rom-Kedar 1988) that

$$
\ln \lambda(p, m, t)=\beta(p, m)+\chi(p, m, t)+O\left(\frac{1}{t^{2}}\right) \text { for } t>t_{0},
$$

where $t_{0}$ is the time required for $p$ to be entrained and then escape from the chaotic region, $\beta(p, m)$ is the long time mean of $\ln \lambda(p, m, t)$ and $\chi(p, m, t)$ is a periodic function in $t$ with zero mean and is a result of the oscillating strain-rate field that persists far from the vortex pair. This behaviour is demonstrated in figure 22 where we show $\mathrm{ln}$ $\lambda$ and $\dot{\lambda} / \lambda$ for a particle initially in the chaotic region and escaping after 12 cycles. Also shown is $\dot{\lambda} / \lambda$ averaged over each period to filter out the $\mathcal{\chi}$-component. 

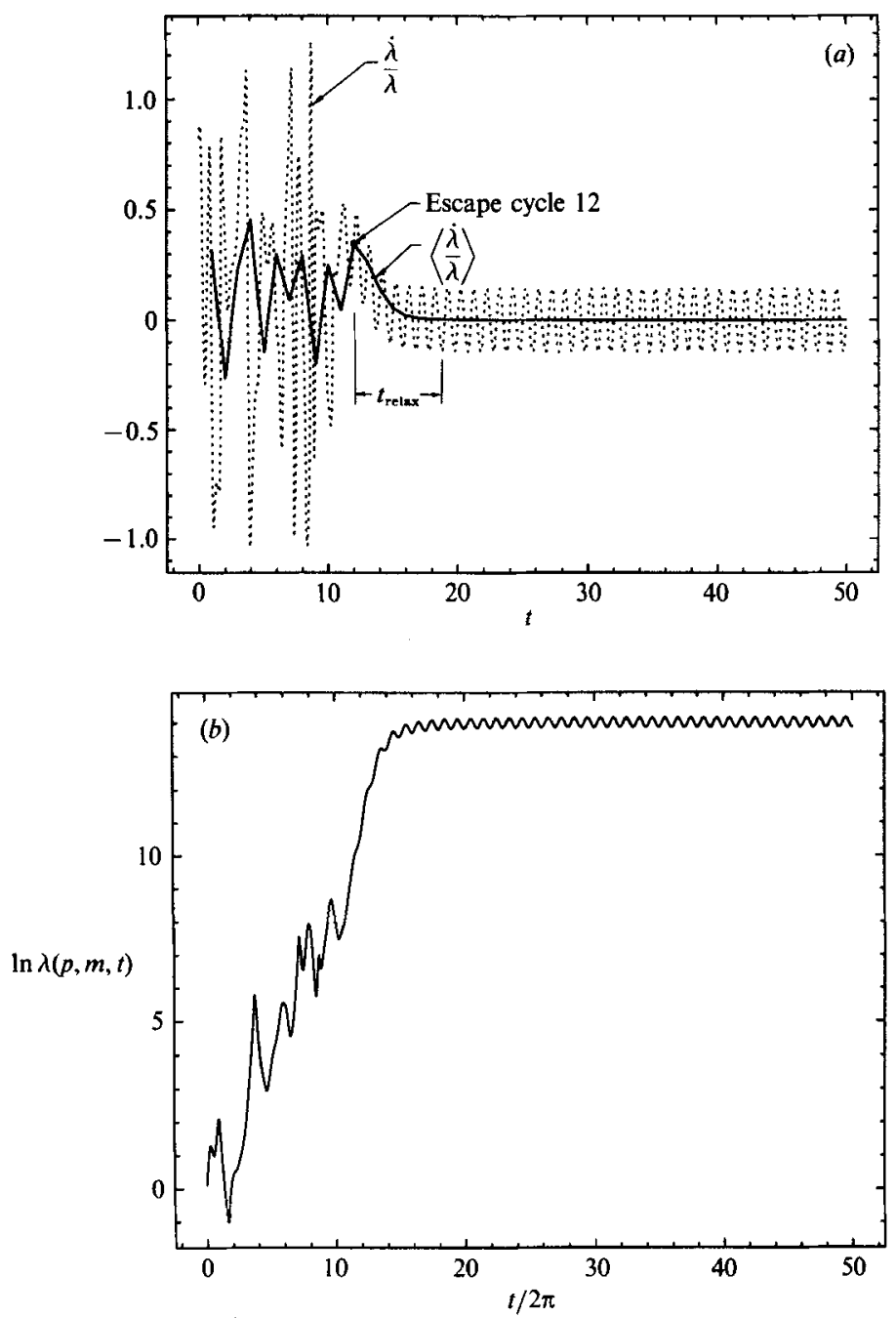

Fraure 22. (a) $\dot{\lambda} / \lambda$ for an initial condition in $A-C ;(b) \ln \lambda$ for the same initial condition.

Thus the Liapunov exponent given by (8.4) is identically zero for almost all particles and, in OVP flow, $\beta$ of (8.5) remains as the useful physical quantity which measures the total elongation of a line element. In particular we shall concentrate on the maximal elongation of an infinitesimal neighbourhood around $p$ with exponent given by

$$
\bar{\beta}(\boldsymbol{p})=\max _{\boldsymbol{m}} \beta(\boldsymbol{p}, \boldsymbol{m}) .
$$

Physically, an infinitesimal dye blob of radius $|m|$ placed at $p$ at $t=0$ will have its length amplified by $\exp (\bar{\beta})$ while it is in or near the chaotic region and, from then on, its length will oscillate periodically with mean $\exp (\bar{\beta})|m|$. Note that we need to maximize $\beta$ over $\boldsymbol{m}$ in contrast to the procedure for finding the largest Liapunov exponent, where almost all vectors will stretch at the same rate eventually. This is the result of analysing the finite time elongation instead of the asymptotic result. The method to obtain $\bar{\beta}$ follows. 
Let $M(t)$ be the fundamental solution matrix of $(8.2)$ so that

$$
\dot{M}=D_{F}(p(t), t) M, \quad M(\theta)=I .
$$

Then, a general solution $\boldsymbol{m}(t)$ of $(8.2)$ is given by $\boldsymbol{m}(t)=\boldsymbol{M}(t) \boldsymbol{m}$. Therefore,

$$
\max _{\boldsymbol{m}} \lambda(\boldsymbol{p}, \boldsymbol{m}, t)=\max _{\boldsymbol{m}}\left(\frac{\boldsymbol{m}^{\mathrm{T}} \boldsymbol{M}^{\mathrm{T}} \boldsymbol{M} \boldsymbol{m}}{\boldsymbol{m}^{\mathrm{T}} \boldsymbol{m}}\right)^{\frac{1}{2}}=\left[\rho\left(\boldsymbol{M}^{\mathrm{T}} \boldsymbol{M}\right)\right]^{\frac{1}{2}}
$$

where $\rho\left(\boldsymbol{M}^{\mathrm{T}} \boldsymbol{M}\right)$ is the maximal eigenvalue of $\boldsymbol{M}^{\mathrm{T}} \boldsymbol{M}$. (Note that in general $\left.\left[\rho \boldsymbol{M}^{\mathbf{T}} \boldsymbol{M}\right]^{\frac{1}{2}} \neq \rho \boldsymbol{M}\right)$, see Goldhirsch, Sulem \& Orszag 1987). To compute $\rho\left(\boldsymbol{M}^{\mathbf{T}} \boldsymbol{M}\right)$ we develop an ODE for the components of $M M^{\mathrm{T}}$, noting that in two dimensions $\rho\left(\boldsymbol{M}^{\mathrm{T}} \boldsymbol{M}\right)=\rho\left(\boldsymbol{M}^{\mathrm{T}}\right)$.

Since the OVP flow is irrotational and incompressible the matrix $D_{F}(p(t), t)$ is symmetric and has the form

$$
\boldsymbol{D}_{F}(\boldsymbol{p}(t), t)=\left(\begin{array}{rr}
a(t) & b(t) \\
b(t) & -a(t)
\end{array}\right)
$$

where

$$
a(t)=\frac{\partial u}{\partial x}(\boldsymbol{p}(t), t), \quad b(t)=\frac{\partial u}{\partial y}(\boldsymbol{p}(t), t)
$$

Using (8.6) and the above form of $\boldsymbol{D}_{F}$ we obtain

$$
\frac{\mathrm{d}}{\mathrm{d} t}\left(\boldsymbol{M} \boldsymbol{M}^{\mathrm{T}}\right)=\frac{\mathrm{d} \boldsymbol{M}}{\mathrm{d} t} \boldsymbol{M}^{\mathrm{T}}+\boldsymbol{M} \frac{\mathrm{d} \boldsymbol{M}^{\mathrm{T}}}{\mathrm{d} t}=\left(\boldsymbol{M} \boldsymbol{M}^{\mathrm{T}}\right) \boldsymbol{D}_{F}+D_{F}\left(\boldsymbol{M} \boldsymbol{M}^{\mathrm{T}}\right)
$$

Now $M M^{T}$ is a symmetric matrix of the form

$$
M M^{\mathbf{T}}=\left(\begin{array}{ll}
q & r \\
r & s
\end{array}\right)
$$

and (8.7), written in component form, gives a system of three ODEs for the matrix elements $q, r, s$. Since $\operatorname{det}\left(\boldsymbol{M} \boldsymbol{M}^{\mathbf{T}}\right)=1$ we obtain the following expression for $\rho\left(\boldsymbol{M M}^{\mathbf{T}}\right)$ :

$$
\rho\left(\boldsymbol{M} \boldsymbol{M}^{\mathrm{T}}\right)=\frac{1}{2}\left\{q+s+\left[(q+s)^{2}-4\right]^{\frac{1}{2}}\right\} .
$$

The quantity $q+s$ can be obtained either from the ODEs for $q, r$ and $s$ or by solving the integral equation

$$
(q+s)^{2}=4\left[\int_{0}^{t} a\left(t^{\prime}\right)(q+s)\left(t^{\prime}\right) \mathrm{d} t^{\prime}\right]^{2}+4\left[\int_{0}^{t} b\left(t^{\prime}\right)(q+s)\left(t^{\prime}\right) \mathrm{d} t^{\prime}\right]^{2}+4
$$

Using the former technique we have found $\bar{\beta}$ for a sample of 530 initial conditions in region $A \cap \bigcup_{k=0}^{\infty} D_{-k}$. The results are presented in figure 23 where we plot $\bar{\beta}$ versus the escape cycle of $p$. Though $\beta$ takes on a range of values for each escape cycle, the general tendency of $\bar{\beta}$ to increase with the escape cycle, as expected, is clear. In figure 24 , we show the average of $\bar{\beta}$ over the set of initial conditions having the same escape cycle. The results indicate that the average stretching rate is correlated with residence time, namely, a longer residence time implies a lower average stretching rate.

To summarize, we have shown that the Liapunov exponents vanish in the OVP flow in the mixing region. This is a result of the flow being open with localized chaos, allowing fluid particles to be convected to infinity after a finite amount of stretching. Therefore we use the total stretch, $\exp (\bar{\beta})$, the elongation of a fluid element while in or near the chaotic region, to quantify the chaos. We found that, on average, this 


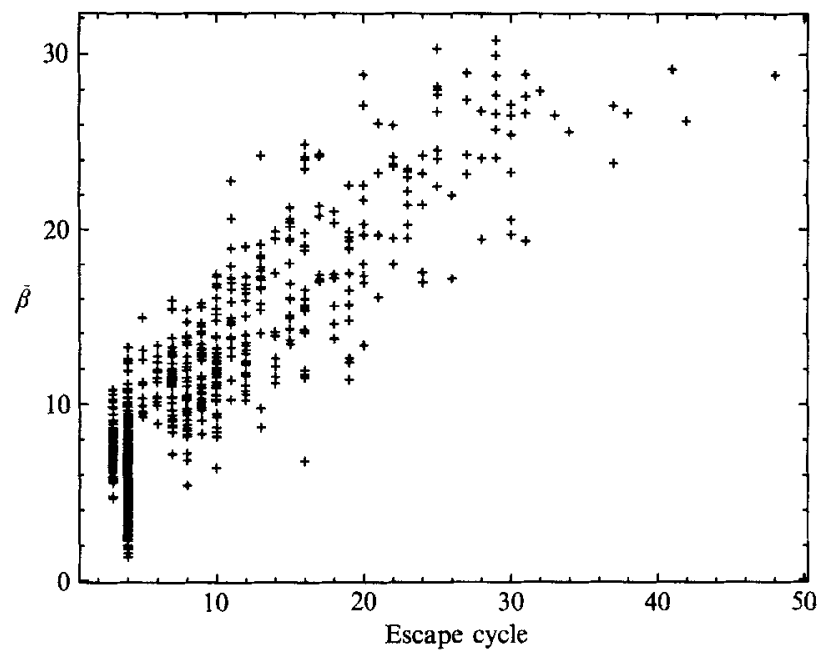

Fraure 23. The total stretch $\bar{\beta}$

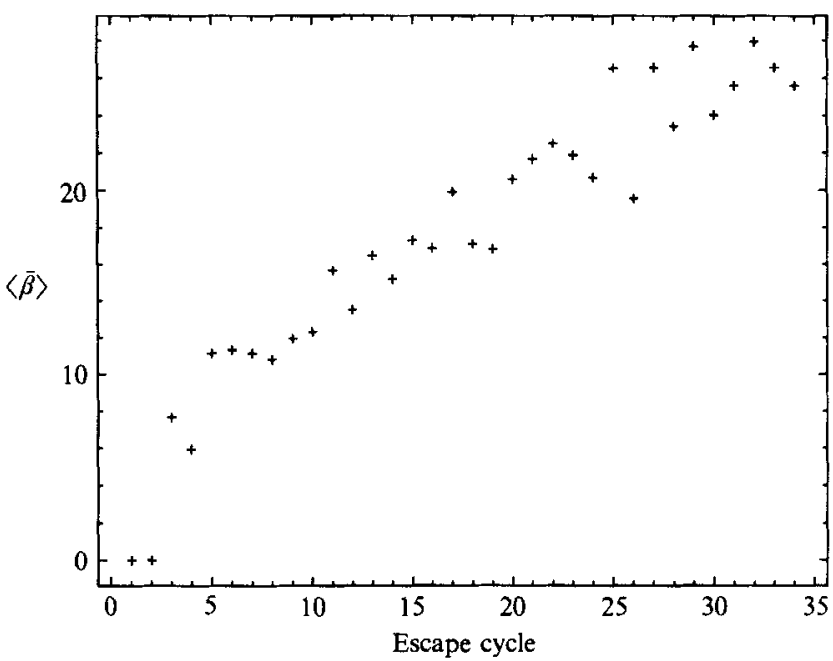

Figure 24. The total stretch averaged over a sample of initial conditions with the same escape cycle.

quantity increases with residence time but that the average stretching rate tends to decrease with residence time.

\section{Summary and conclusions}

We have investigated the flow governed by a vortex pair in the presence of an external strain-rate field that oscillates sinusoidally in time. In particular, we studied transport and mixing of passive particles in this flow. The flow depends on two dimensionless parameters - the period of the oscillation divided by the time required for the vortex pair to travel a distance equal to their separation and the strain-rate amplitude divided by the oscillation frequency.

If the amplitude of the external field is zero then, in a frame moving with the vortex pair, the flow is steady. A fixed, closed volume of fluid is trapped and moves 
with the vortex pair for all time. This volume of fluid or 'bubble' is bounded by two limiting streamlines that connect at two hyperbolic stagnation points, one on the upstream side of the bubble and one on the downstream side. No entrainment or detrainment takes place into or out of this volume. Mixing is poor as two particles an infinitesimal distance apart only separate at most linearly in time for large times.

If the strain-rate amplitude is non-zero, the flow is time periodic in a frame moving with the average speed of the vortex pair and each vortex moves on a closed orbit in this frame. However, some particle motions are quite complicated. During each cycle of the oscillation a certain volume of fluid that approaches from the upstream side is entrained into the fluid bubble moving with the vortex pair. A particle within this entrained fluid volume moves chaotically during its time of residence in the comoving bubble. These particles subsequently escape (are detrained) according to a discrete distribution over the number of cycles in residence till escape. Another distribution of residence times, that corresponding to those particles initially in the bubble at cycle zero, is easily computed from the former distribution.

A quantitative understanding of this transport mechanism was best achieved by examining the Poincaré map for the particle motion. In particular, we considered the stable and unstable manifolds of the two hyperbolic fixed points of the map. For the unperturbed flow, the fixed points coincide with the stagnation points in the flow. Similarly, the unstable manifolds of the upstream fixed point and the stable manifolds of the downstream fixed point coincide with the limiting streamlines in the unperturbed flow and, therefore, coincide with each other. For the perturbed flow, these manifolds break apart and intersect each other transversally, forming a tangle. Within this tangle there are two infinite families of lobes with the boundary of each lobe consisting of a segment of the unstable manifold and, the remainder, a segment of the stable manifold. Any given lobe generates all other lobes in the same family by mapping backwards and forward in time.

One lobe in particular will be entrained during the next cycle and, in the other family, there is a lobe that will be detrained. By incompressibility all lobes have the same volume and it is this amount of fluid therefore that is entrained and detrained during each cycle. Lobes within a given family do not intersect one another. However, lobes from one family intersect members from the other family. The areas or volumes of intersection correspond directly to the residence time distributions mentioned above. Thus we have a tangle dynamics. Namely, all information concerning transport or dispersion of particles in this two-space plus time-dependent flow field is generated by one-dimensional objects - the stable and unstable manifolds of the fixed points of the Poincare map.

The above results do not require that the strain-rate amplitude be small. However, if the amplitude is small we used regular perturbation theory in the form of the Melnikov technique to check for the existence of transversal intersections and to estimate the width of the fluid zone along the original dividing streamline that participates in the exchange process. In addition we have shown that the lobe area is proportional to the integral of the Melnikov function over one cycle. This analysis showed that, as the period of oscillation increases from zero, the entrainment rates rises from zero to a maximum then falls to zero and rises again.

For arbitrary values of the strain-rate amplitude the lobe structure may be computed numerically. This is a relatively simple matter because the unstable manifolds are attractors in forward time and the stable manifolds are attractors integrating backwards in time. Of course, as one attempts to follow a given lobe for 
a large number of cycles a rapidly increasing number of points is required to define the structure of the lobe boundary because of the chaotic motion of the boundary points that remain in the bubble. Lobe intersection volume may be computed by tracking lobe boundaries as described above but as an alternative method, and the one used in this paper, one can simply track a uniform array of closely spaced points that initially fill only the lobe that will be entrained during the next cycle.

We have shown that the existence of transverse heteroclinic orbits, i.e. the lobe structures, give rise to Smale horseshoes as a result of a stretching and folding mechanism present in the Poincaré map. These horseshoes represent the underlying mechanism for chaotic particle motion. To quantify this chaotic motion and, in particular, the rate of stretching of material elements, we investigated the total elongation of an infinitesimal material element and its dependence on time spent within the bubble.

The concepts and analysis discussed in this paper should be useful in a wide variety of applications. Further development might concentrate on (1) extension of the present results for time-periodic flows to quasi-periodic or chaotic fluid flows, (2) connecting the present work to coarse-grained approaches to turbulent transport using convection-diffusion equations, and (3) developing analytical techniques for the lobe intersection problem by, for example, deriving appropriate one-dimensional maps.

This work was supported in part by Caltech's Program in Advanced Technologies, sponsored by Aerojet General, General Motors, and TRW, and by the Air Force Office of Scientific Research through the Jet Propulsion Laboratory. V.R. Acknowledges the support of a Zonta Amelia Earhart Fellowship and of a PEO International Peace Scholarship.

\section{Appendix A. Vortex pair in a wavy-walled channel}

We show that the particle motion given by (2.3) and (2.5) approximates the motion in the vicinity of a vortex pair moving in a wavy-walled channel. Consider the following solution to the Euler equations, given by the stream function of a vortex pair, $\Psi_{\mathrm{v}}$, plus the stream function of a potential flow:

$$
\Psi=\Psi_{\mathrm{v}}+\Psi_{\mathrm{pot}},
$$

where

$$
\Psi_{\mathrm{v}}=-\frac{\Gamma}{4 \pi} \log \frac{\left(x-x_{\mathrm{v}}\right)^{2}+\left(y-y_{\mathrm{v}}\right)^{2}}{\left(x-x_{\mathrm{v}}\right)^{2}+\left(y+y_{\mathrm{v}}\right)^{2}}
$$

and

$$
\Psi_{\mathrm{pot}}=\left(V+\epsilon V_{\mathbf{1}}(\epsilon)\right) y-\frac{\epsilon}{k^{2}} \cos (k x) \sinh (k y) .
$$

Here $\pm \Gamma$ are the circulations of the vortices whose positions are $\left(x_{\mathrm{v}}(t), \pm y_{\mathrm{v}}(t)\right)$; $V+\epsilon V_{1}(\epsilon)$ is the average fluid velocity in the channel far away from the vortex pair and $\epsilon V_{1}(\epsilon)$ is defined such that the average velocity of the vortex pair is independent of $\epsilon$ (see below). The vortices move with the fluid velocity given by

$$
\frac{\mathrm{d} x}{\mathrm{~d} t}=\frac{\partial \Psi}{\partial y}, \quad \frac{\mathrm{d} y}{\mathrm{~d} t}=-\frac{\partial \Psi}{\partial x}
$$

For $\epsilon=0$, we have

$$
x_{\mathrm{v}}(t)=\left(V+\frac{\Gamma}{4 \pi d}\right) t, \quad y_{\mathrm{v}}(t)=d .
$$


Setting $\Psi=\Psi_{\text {wall }}=$ const in (A $1 a$ ) and assuming that $y_{\mathrm{v}} / y_{\text {wall }} \ll 1$ we find the equation for the wall boundary,

$$
\begin{aligned}
y & =y_{\text {wall }}(x) \\
& =\frac{\Psi_{\text {wall }}}{V}-\frac{\epsilon V_{1}(0) \Psi_{\text {wall }}}{V^{2}}+\frac{\epsilon}{k^{2} V} \sinh \left(\frac{k \Psi_{\text {wall }}}{V}\right) \cos (k x)+O\left(\epsilon^{2}\right)+O\left(\frac{y_{\mathrm{v}}}{y_{\text {wall }}}\right),
\end{aligned}
$$

confirming that (A 1) represent flow in a wavy-walled channel where the walls are sufficiently far from the vortices.

Now we compute particle motion by (A 2$)$ and let $x=\tilde{x}+f(t), x_{\mathrm{v}}(t)=\tilde{x}_{\mathrm{v}}(t)+f(t)$. If $k \tilde{x}_{\mathrm{v}} \ll 1$ and we consider the flow field near the vortices with $k y \ll 1$, then we obtain the desired form:

$$
\begin{aligned}
& \frac{\mathrm{d} \tilde{x}}{\mathrm{~d} t}=-\frac{\Gamma}{2 \pi}\left[\frac{\left(y-y_{\mathrm{v}}\right)}{\left(\tilde{x}-\tilde{x}_{\mathrm{v}}\right)^{2}+\left(y-y_{\mathrm{v}}\right)^{2}}-\frac{y+y_{\mathrm{v}}}{\left(\tilde{x}-\tilde{x}_{\mathrm{v}}\right)^{2}+\left(y+y_{\mathrm{v}}\right)^{2}}\right]-V_{\mathrm{v}}+\epsilon \tilde{x} \sin (\omega t)+O\left(\epsilon^{2}\right), \\
& \frac{\mathrm{d} y}{\mathrm{~d} t}=\frac{\Gamma\left(\tilde{x}-x_{\mathrm{v}}\right)}{2 \pi}\left[\frac{1}{\left(\tilde{x}-\tilde{x}_{\mathrm{v}}\right)^{2}+\left(y-y_{\mathrm{v}}\right)^{2}}-\frac{1}{\left(\tilde{x}-\tilde{x}_{\mathrm{v}}\right)^{2}+\left(y+y_{\mathrm{v}}\right)^{2}}\right]-\epsilon y \sin (\omega t)+O\left(\epsilon^{2}\right)
\end{aligned}
$$

if $f(t)$ is the solution to

$$
\frac{\mathrm{d} f}{\mathrm{~d} t}=-\frac{\epsilon}{k} \cos (k f(t))+V+\frac{\Gamma}{4 \pi d}+\epsilon^{2} V_{2}(\epsilon),
$$

where $V_{2}(\epsilon)$ is defined such that

$$
f(t)=\left(V+\frac{\Gamma}{4 \pi d}\right) t+\epsilon g(l, \epsilon)
$$

where $g$ is periodic in $t$. We also have that

$$
\begin{gathered}
\omega=k\left(V+\frac{\Gamma}{4 \pi d}\right) \\
V_{\mathrm{v}}=\frac{\Gamma}{4 \pi d}-\epsilon V_{1}(\epsilon) .
\end{gathered}
$$

\section{Appendix B. Expansion of the equations of motion}

The non-dimensional equations of motion (2.3) have the following expansion in $\epsilon$ :

$$
\begin{aligned}
& \frac{\mathrm{d} x}{\mathrm{~d} t}=f_{1}(x, y)+\epsilon g_{1}(x, y, t / \gamma ; \gamma)+O\left(\epsilon^{2}\right), \\
& \frac{\mathrm{d} y}{\mathrm{~d} t}=f_{2}(x, y)+\epsilon g_{2}(x, y, t / \gamma ; \gamma)+O\left(\epsilon^{2}\right),
\end{aligned}
$$

where the $f_{i}$ are given by

$$
f_{1}=-\frac{y-1}{I_{-}}+\frac{y+1}{I_{+}}-\frac{1}{2}, \quad f_{2}=x\left[\frac{1}{I_{-}}-\frac{1}{I_{+}}\right],
$$


and the $g_{i}$ are given by

$$
\begin{aligned}
& \begin{aligned}
g_{1}=[\cos (t / \gamma)-1]\left\{\frac{1}{I_{-}}+\frac{1}{I_{+}}-\frac{2(y-1)^{2}}{I_{-}^{2}}\right. & \left.-\frac{2(y+1)^{2}}{I_{+}^{2}}\right\} \\
+ & +\frac{x}{\gamma} \sin (t / \gamma)\left\{\gamma^{2}\left[\frac{y-1}{I_{-}^{2}}-\frac{y+1}{I_{+}^{2}}\right]+1\right\}-\frac{1}{2}, \\
g_{2}=2 x[\cos (t / \gamma)-1]\left\{\frac{y-1}{I_{-}^{2}}+\frac{y+1}{I_{+}^{2}}\right\} & +\frac{1}{\gamma} \sin (t / \gamma) \\
& \times\left\{\frac{\gamma^{2}}{2}\left[\frac{1}{I_{-}}-\frac{1}{I_{+}}\right]-x^{2} \gamma^{2}\left[\frac{1}{I_{-}^{2}}-\frac{1}{I_{+}^{2}}\right]-y\right\} .
\end{aligned} \\
& \text { The definitions of } I_{ \pm} \text {are } \quad I_{ \pm}=x^{2}+(y \pm 1)^{2}
\end{aligned}
$$

\section{Appendix C. The Melnikov function}

In this Appendix, we discuss some aspects of the Melnikov function. Specifically, we shall discuss how it arises and what it measures. Recall that the perturbed velocity field can be written in the form

$$
\dot{x}=f_{1}(x, y)+\epsilon g_{1}(x, y, \theta ; \gamma)+O\left(\epsilon^{2}\right), \quad \dot{y}=f_{2}(x, y)+\epsilon g_{2}(x, y, \theta, \gamma)+O\left(\epsilon^{2}\right), \quad \dot{\theta}=1 / \gamma
$$

As a convenient shorthand notation we shall often write (C 1) in the following vector form :

$$
\dot{q}=f(q)+\epsilon g(q, \theta ; \gamma)+O\left(\epsilon^{2}\right), \quad \dot{\theta}=1 / \gamma,
$$

where $\boldsymbol{q}=(x, y), \boldsymbol{f}=\left(f_{1}, f_{2}\right)$, and $\boldsymbol{g}=\left(g_{1}, g_{2}\right)$.

In each case the unperturbed velocity field is obtained by taking $\epsilon=0$ in (C 1) and (C 2). We study the two-dimensional Poincaré map $T$ obtained from the solutions of (C 2) which is defined as follows:

$$
\begin{gathered}
T: \Sigma \rightarrow \Sigma \\
(x(0), y(0)) \mapsto(x(2 \pi \gamma), y(2 \pi \gamma)) .
\end{gathered}
$$

Recall that the Poincare map obtained from the unperturbed velocity field has saddle points at $p_{+}$and $p_{-}$which are connected to each other by the three heteroclinic orbits $\Psi_{\mathrm{u}}, \Psi_{0}$, and $\Psi_{\ell}$ (see figure 1). As noted earlier, by symmetry of the unperturbed flow $\Psi_{0}$ remains unbroken under the external strain. We use the Melnikov function to determine the behaviour of $\Psi_{\mathrm{u}}$ and $\Psi_{\ell}$. Since the perturbed velocity field is symmetric about the $x$-axis, for definiteness, we shall only draw pictures of the upper half-plane in our development of the Melnikov function.

The construction of the Melnikov function consists of four steps: (1) develop a parametrization of the unperturbed heteroclinic orbit in the Poincare section; (2) define a moving coordinate system along the unperturbed heteroclinic orbit in the Poincaré section; (3) define the distance between $W_{+, \epsilon}^{\mathrm{s}}$ and $W_{-, \varepsilon}^{\mathrm{u}}$ in the moving coordinate system at points along the unperturbed heteroclinic orbit; (4) utilize Melnikov's trick to develop a computable form for the geometrically defined distance between $W_{+, \epsilon}^{\mathrm{s}}$ and $W_{-, \epsilon}^{\mathrm{u}}$ of the points along the unperturbed heteroclinic orbit.

We begin with step (1).

Step (1). Let $q_{\mathrm{u}}(t)$ denote a heteroclinic trajectory of the unperturbed velocity field 
which lies in $\Psi_{u}$. Then, since the unperturbed velocity field is not time dependent (i.e. it is autonomous), $\boldsymbol{q}_{\mathrm{u}}\left(t-t_{0}\right)$ is also a heteroclinic trajectory of the unperturbed velocity field which lies in $\Psi_{1}$ for any $t_{0} \in E$ (see Arnol'd 1973 for a proof of this fact). Thus $\boldsymbol{q}_{\mathrm{u}}\left(-t_{0}\right), t_{0} \in R$ provides a parametrization of $\Psi_{\mathrm{u}}$ where $t_{0}$ is the unique time that it takes for a fluid particle on $\Psi_{\mathrm{u}}$ to flow to $q_{\mathrm{u}}(0)$.

Step (2). The vector $f^{\perp}\left(q_{\mathrm{u}}\left(-t_{0}\right)\right)=\left(-f_{\mathrm{2}}\left(\boldsymbol{q}_{\mathrm{u}}\left(-t_{0}\right)\right), f_{1}\left(\boldsymbol{q}_{\mathrm{u}}\left(-t_{0}\right)\right)\right.$ is perpendicular to $\Psi_{\mathrm{u}}$ at each point $q_{\mathrm{u}}\left(-t_{0}\right)$ on $\Psi_{\mathrm{u}}$. Thus varying $t_{0}$ will serve to move $\boldsymbol{f}^{\perp}\left(\boldsymbol{q}_{\mathrm{u}}\left(-t_{0}\right)\right)$ along $\Psi_{\mathrm{u}}$ and the distance between $W_{+, \epsilon}^{\mathrm{s}}$ and $W_{-, \epsilon}^{\mathrm{u}}$ will be measured along $f^{\perp}\left(q_{\mathrm{u}}\left(-t_{0}\right)\right)$.

Step (3). At $\epsilon=0, W_{+}^{\mathrm{s}}$ and $W_{-}^{\mathrm{s}}$ intersect $f^{\perp}\left(\boldsymbol{q}_{\mathbf{u}}\left(-t_{0}\right)\right)$ transversely at each $\boldsymbol{q}_{\mathrm{u}}\left(-t_{0}\right) \in \Psi_{\mathrm{u}}$ (see Arnol'd 1982 for a definition of the transversal intersection of two manifolds). The intersections are preserved under perturbations so that for $\epsilon$ sufficiently small $W_{+, \varepsilon}^{\mathrm{s}}$ and $W_{-, \varepsilon}^{\mathrm{u}}$ intersect $f^{\perp}\left(\boldsymbol{q}_{\mathbf{u}}\left(-t_{0}\right)\right)$ transversely in the points $\boldsymbol{q}_{\mathrm{f}}^{\mathrm{s}}$ and $\boldsymbol{q}_{\varepsilon}^{\mathrm{u}}$. Thus we define the distance between $W_{+, \varepsilon}^{\mathrm{s}}$ and $W_{-, \varepsilon}^{\mathrm{u}}$ at the point $\boldsymbol{q}_{\mathrm{u}}\left(-t_{0}\right)$ to be

$$
\text { distance }=\left|\boldsymbol{q}_{e}^{\mathrm{u}}-\boldsymbol{q}_{\epsilon}^{\mathrm{s}}\right| \text {. }
$$

see figure 25. The problem with this definition of the distance is that is does not lend itself to an expression which can easily be computed without solving explicitly for fluid particle motions of the perturbed velocity field; a task which would be quite formidable. However, following Melnikov (1963), we define the following 'signed' distance measurement:

$$
d\left(t_{0}, \epsilon\right)=\frac{f^{\perp}\left(q^{\mathrm{u}}\left(-t_{0}\right)\right) \cdot\left(\boldsymbol{q}_{\epsilon}^{\mathrm{u}}-\boldsymbol{q}_{\epsilon}^{\mathrm{s}}\right)}{\left\|f\left(\boldsymbol{q}^{\mathrm{u}}\left(-t_{\mathbf{0}}\right)\right)\right\|}
$$

where - denotes the usual vector dot product. It should be clear that, by the choice of $\boldsymbol{q}_{\epsilon}^{\mathrm{u}}$ and $\boldsymbol{q}_{\epsilon}^{\mathrm{s}}, d\left(t_{\mathbf{0}}, \epsilon\right)=0$ if and only if $\boldsymbol{q}_{\epsilon}^{\mathrm{e}}=\boldsymbol{q}_{\epsilon}^{\mathrm{s}}$.

Now because $W_{+, \epsilon}^{\mathrm{s}}$ and $W_{-, \epsilon}^{\mathrm{u}}$ vary differentially with respect to parameters (Fenichel 1971; Hirsch, et al. 1977) we can Taylor expand (C 5) about $\epsilon=0$ to obtain

$$
d\left(t_{0}, \epsilon\right)=\epsilon \frac{f^{\perp}\left(\boldsymbol{q}_{\mathrm{u}}\left(-t_{0}\right)\right) \cdot\left(\left.\frac{\partial \boldsymbol{q}^{\mathrm{u}}}{\partial \epsilon}\right|_{\epsilon=0}-\left.\frac{\partial \boldsymbol{q}^{\mathrm{s}}}{\partial \epsilon}\right|_{\epsilon=0}\right)}{\left\|\boldsymbol{f}\left(\boldsymbol{q}_{\mathrm{u}}\left(-t_{0}\right)\right)\right\|}+O\left(\epsilon^{\mathbf{2}}\right),
$$

where we have used the fact that $\boldsymbol{q}_{0}^{\mathrm{u}}=\boldsymbol{q}_{0}^{\mathrm{s}}$.

The Melnikov function, denoted $M\left(t_{0}\right)$, is defined to be

$$
M\left(t_{0}\right)=f^{\perp}\left(\boldsymbol{q}_{\mathbf{u}}\left(-t_{0}\right)\right) \cdot\left(\left.\frac{\partial \boldsymbol{q}_{\varepsilon}^{\mathrm{u}}}{\partial \epsilon}\right|_{\epsilon=0}-\left.\frac{\partial \boldsymbol{q}_{\epsilon}^{\mathrm{s}}}{\partial \epsilon}\right|_{\epsilon=0}\right)
$$

and is (up to the normalization factor $\left\|f\left(q_{\mathrm{u}}\left(-t_{0}\right)\right)\right\|^{-1}$ ) the leading-order term in the Taylor series expansion for the distance between $W_{+, \epsilon}^{\mathrm{s}}$ and $W_{-, \epsilon}^{\mathrm{u}}$ at the point $q_{\mathrm{u}}\left(-t_{0}\right)$.

Step (4). Melnikov (1963) was able to derive an expression for (C 7) without explicitly computing particle paths of the perturbed velocity field. His procedure consisted of the following steps. (a) Prove that the particle paths of the perturbed velocity field through the points $\boldsymbol{q}_{\epsilon}^{\mathrm{u}}$ and $\boldsymbol{q}_{\varepsilon}^{\mathrm{s}}$ exist on the time intervals $(-\infty, 0]$ and $[0, \infty)$, respectively. (b) Using $(a)$ along with the first variational equation for solutions through $\boldsymbol{q}_{\varepsilon}^{\mathrm{u}}$ and $\boldsymbol{q}_{\epsilon}^{\text {s }}$ (i.e. regular perturbation theory) derive a linear firstorder ordinary differential equation for the time-dependent Melnikov function

$$
M\left(t, t_{0}\right)=f^{\perp}\left(\boldsymbol{q}_{\mathrm{u}}\left(t-t_{0}\right)\right) \cdot\left(\left.\frac{\partial \boldsymbol{q}_{\epsilon}^{\mathrm{u}}(t)}{\partial \epsilon}\right|_{\epsilon=0}-\left.\frac{\partial \boldsymbol{q}_{\epsilon}^{\mathrm{s}}(t)}{\partial \epsilon}\right|_{\epsilon=0}\right),
$$

where $\boldsymbol{q}_{\epsilon}^{\mathrm{u}}(t)$ and $\boldsymbol{q}_{\epsilon}^{\mathrm{s}}(t)$ are particle paths of the perturbed velocity field satisfying 


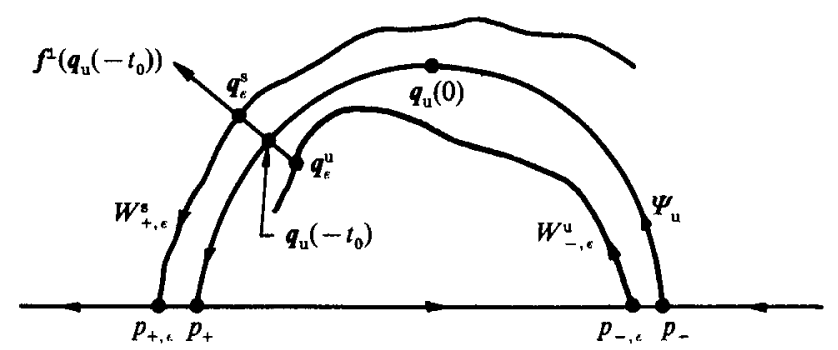

Figure 25. The geometry of the distance between $W_{+, \epsilon}^{\mathrm{s}}$ and $W_{-, \epsilon^{\circ}}$.

$\boldsymbol{q}_{\varepsilon}^{\mathrm{u}}(0)=\boldsymbol{q}_{\mathrm{s}}^{\mathrm{u}}$ and $\boldsymbol{q}_{\epsilon}^{\mathrm{s}}(0)=\boldsymbol{q}_{\varepsilon}^{\mathrm{s}}$, respectively. Thus $M\left(0, t_{0}\right) \equiv M\left(t_{0}\right)$. (c) Solve the linear firstorder differential equation for $M\left(t, t_{0}\right)$ and obtain the Melnikov function by evaluating at $t=0$. In the process boundary conditions for the solution at $\pm \infty$ are imposed which were the reason for needing the existence proof of particle paths on semiinfinite time intervals as described in $(a)$.

For the full details of these steps see Guckenheimer \& Holmes (1983) or Wiggins (1988). Finally, one obtains the following form for the Melnikov function:

$$
\boldsymbol{M}\left(t_{0}\right)=\int_{-\infty}^{\infty}\left[f_{1}\left(\boldsymbol{q}_{\mathrm{u}}(t)\right) g_{2}\left(\boldsymbol{q}_{\mathrm{u}}(t), t+t_{0}\right)-f_{2}\left(\boldsymbol{q}_{\mathrm{u}}(t)\right) g_{1}\left(\boldsymbol{q}_{\mathrm{u}}(t), t+t_{0}\right)\right] \mathrm{d} t
$$

and we have the following key theorem.

Theorem C 1. Suppose there exists $t_{0}=\bar{t}_{0}$ such that

$$
\text { (1) } M\left(\bar{t}_{0}\right)=0, \quad \text { (2) } \frac{\partial M}{\partial t_{0}}\left(\bar{t}_{0}\right) \neq 0
$$

Then $W_{+, \varepsilon}^{\mathrm{s}}$ and $W_{-, \epsilon}^{\mathrm{u}}$ intersect transversely near $\boldsymbol{q}_{\mathrm{u}}\left(-\bar{t}_{0}\right)$. If $M\left(t_{0}\right)$ is bounded away from zero for all $t_{0}$, then $W_{+, e}^{\mathrm{s}}$ and $W_{-, e}^{\mathrm{u}}$ are bounded away from each other.

Proof. See Guckenheimer \& Holmes (1983) or Wiggins (1988).

Thus we can determine whether or not $W_{+, \epsilon}^{\mathrm{s}}$ and $W_{-, \epsilon}^{\mathrm{u}}$ intersect without solving for fluid particle motions of the perturbed velocity field.

We now point out two properties of the Melnikov function which are important.

1. Zeros of $M\left(t_{0}\right)$ correspond to primary intersection points. The Melnikov function is a first-order measure of the distance between $W_{+, \epsilon}^{\mathrm{s}}$ and $W_{-, \epsilon}^{\mathrm{u}}$ along the line $f^{\perp}\left(q_{u}\left(-t_{0}\right)\right)$. However, it is possible that $W_{+, \varepsilon}^{\mathrm{s}}$ and $W_{-, \epsilon}^{\mathrm{u}}$ may intersect $f^{\perp}\left(q_{\mathrm{u}}\left(-t_{0}\right)\right)$ many times as depicted in figure 26. The question arises of which two points on $W_{+, \varepsilon}^{\mathrm{s}} \cap f^{\perp}\left(q_{\mathrm{u}}\left(-t_{0}\right)\right)$ and $W_{-, \varepsilon}^{\mathrm{u}} \cap f^{\perp}\left(q_{\mathrm{u}}\left(-t_{0}\right)\right)$ is the distance being measured. The answer to this question comes from the validity of the regular perturbation theory which was used to obtain a computable expression for the Melnikov function (step 4). The fact that we can approximate fluid particle motions of the perturbed velocity field uniformly only on semi-infinite time intervals coupled with the geometry of the timedependent Melnikov function implies that the Melnikov function is a measurement between points in $W_{+, \epsilon}^{\mathrm{s}}$ and $W_{-, \epsilon}^{\mathrm{u}}$ along $f^{\perp}\left(q_{\mathrm{u}}\left(-t^{0}\right)\right)$ which are 'closest' to $p_{+, \epsilon}$ and $p_{-, \epsilon}$, respectively, in the sense of elapsed time of motion along $W_{+, \varepsilon}^{\mathrm{s}}$ and $W_{-, \epsilon}$. These points are denoted $\overline{\boldsymbol{q}}_{\epsilon}^{\mathrm{s}}$ and $\overline{\boldsymbol{q}}_{\epsilon}^{\mathrm{u}}$ in figure 26. From definition 5.1, it follows that $\overline{\boldsymbol{q}}_{\epsilon}^{\mathrm{s}}$ and $\overline{\boldsymbol{q}}_{\epsilon}^{\mathrm{u}}$ are primary intersection points. For more details see Wiggins (1988).

2. The relative displacement of $W_{+, \varepsilon}^{\mathrm{s}}$ and $W_{-, \epsilon}^{\mathrm{u}}$. Since transport in the mixing region is governed by $W_{+, \varepsilon}^{\mathrm{s}}$ and $W_{-, \varepsilon}^{\mathrm{u}}$ it is useful to know their relative positions and, because 


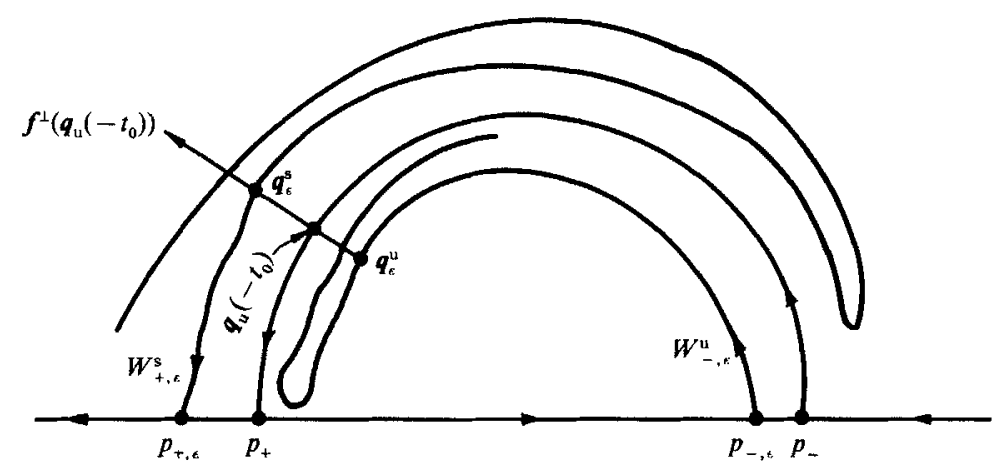

FIGURE 26. Intersection of the manifolds with $f^{\perp}\left(q_{u}\left(-t_{0}\right)\right)$.
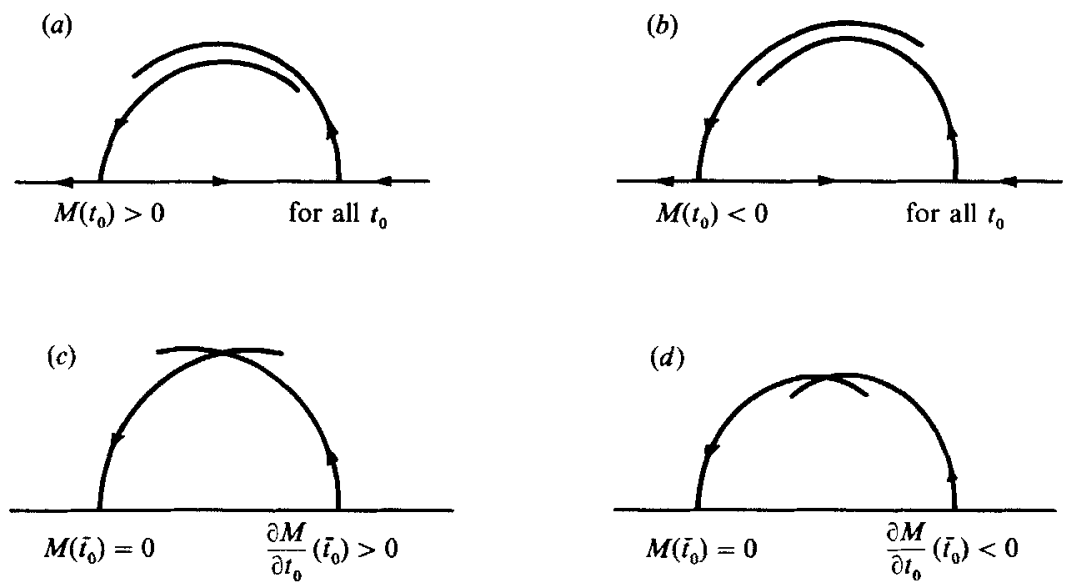

Figure 27. The Melnikov function and the relative orientations of the manifolds.

the Melnikov function is a signed distance measurement it contains this information. From the definition of the distance between $W_{+, \varepsilon}^{\mathrm{s}}$ and $W_{-, \epsilon}^{\mathrm{u}}$ given in (C 5), it is simple to show that the geometry of the manifolds shown in figure 27 holds. For the OVP flow the Melnikov function is given by equation (4.3) and figures 4 and 5 confirm the relation between the Melnikov function and the relative positions of the stable and unstable manifolds for this flow.

3. Periodicity of $M\left(t_{0}\right)$. The Melnikov function is a periodic function of $t_{0}$ having the same period as the external strain field (see Guckenheimer \& Holmes 1983). This is an indication that one heteroclinic point implies the existence of a countable infinity of heteroclinic points.

\section{Appendix D. The Melnikov function and lobe motion}

We now give the proof of Theorem 5.1 which is restated below:

Theorem D 1. Suppose $M\left(t_{0}\right)$ has $2 n$ simple zeros in one period $\tau$. Then

$$
T\left(E_{i}\right)=E_{i+n}, \quad T\left(D_{i}\right)=D_{i+n}
$$


Proof. From Appendix C, simple zeros of the Melnikov function correspond to p.i.p.s. We denote the $2 n$ zeros of $M\left(t_{0}\right)$ in one period as follows:

$$
q_{i}^{E}<q_{i}^{D}<q_{i+1}^{E}<\ldots<q_{i+n-1}^{E}<q_{i+n-1}^{D},
$$

where the notation and ordering (see definition 5.3) are chosen such that $E_{i+k}$ is formed by $q_{i+k}^{E}$ and $q_{i+k}^{D}$ and $D_{i+k}$ is formed by $q_{i+k}^{D}$ and $q_{i+k+1}^{E}$ for $k=1, \ldots, n-1$.

Now by orientation preservation p.i.p.s maintain their relative ordering along $W_{-, \varepsilon}^{a}$ under iteration by $T$ and because the velocity field (and hence the Melnikov function) is periodic in time with period $\tau$ we have

and

$$
\begin{aligned}
& T\left(q_{i}^{E}\right)=q_{i+n}^{E} \\
& T\left(q_{i}^{D}\right)=q_{i+n}^{D}
\end{aligned}
$$

Then by definition it follows that

and

$$
\begin{gathered}
T\left(E_{i}\right)=E_{i+n} \\
T\left(D_{i}\right)=D_{i+n} .
\end{gathered}
$$

\section{Appendix E. Some properties of Poincaré maps}

In this Appendix we shall point out some general properties of Poincaré maps which have fluid dynamical consequences and are not usually discussed in standard texts.

(a) Area preservation. A consequence of the conservation of mass and incompressibility is that the Poincaré map preserves area.

(b) Orientation preservation. Poincaré maps obtained by discretely sampling trajectories of ordinary differential equations have the property of preserving the orientation of area elements. Analytically, this means that the determinant of the Jacobian of the map is strictly positive over its domain of definition (note: by area preservation the determinant of the Jacobian is identically one). Geometrically, orientation preservation can be described as follows. Consider a simply connected area element $D$ with three points denoted $a, b$, and $c$ on the boundary of $D$. Suppose that as one walks along the boundary of $D$ in a counterclockwise sense (i.e. with the left arm pointed toward the interior of $D$ ) beginning at $a$ so that next $b$ and $c$ is encountered. Now let $T_{\bar{\theta}}(D)=D^{\prime}$ with $T_{\bar{\theta}}(a)=a^{\prime}, T_{\bar{\theta}}(b)=b^{\prime}$, and $T_{\bar{\theta}}(c)=c^{\prime}$. $T_{\bar{\theta}}$ is orientation preserving if as one walks along the boundary of $D^{\prime}$ in a counterclockwise sense starting at $a^{\prime}$ then next $b^{\prime}$ and then $c^{\prime}$ is encountered. This implies that the interior of a closed curve is mapped to the interior of its image. See figure 28 for an illustration of the geometry of orientation preservation.

(c) Variation of the cross-section $\sum^{\bar{\theta}}$. Notice from (3.2) that the Poincare map depends on the phase of the strain-rate field. The question then arises as to how the Poincare map changes as the phase of the field is varied? Fortunately, there is no qualitative difference in any of these maps. The technical term is that the different maps are differentiably equivalent (see Irwin 1980) which means that given any two Poincaré maps obtained by fixing two different phases of the strain-rate field there exists a differentiable change of coordinates which transforms one map into the other. In particular, the nature of the stability of a fluid particle trajectory is the same for each Poincaré map. Since there is no qualitative difference in the Poincaré maps we shall take $\bar{\theta}=0$. This choice has the advantage that the Poincare map on this cross-section is symmetric about the $y$-axis with time reversed. We refer to the associated Poincaré map as $T$. 

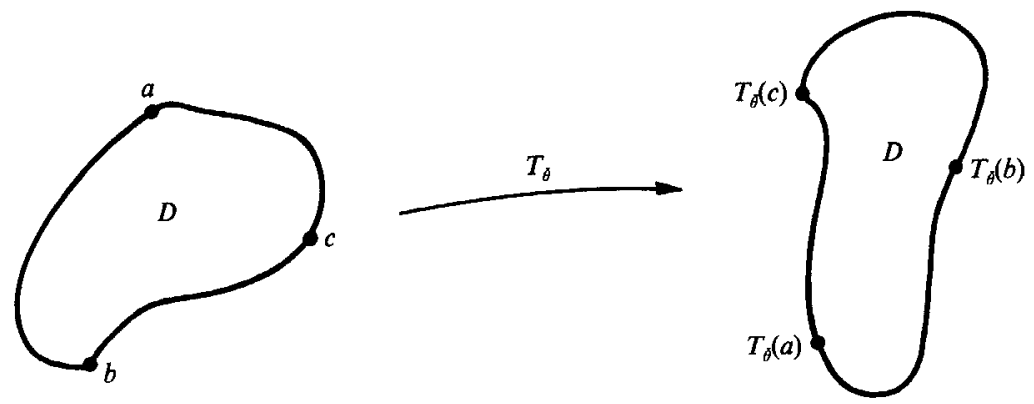

FIGURE 28. Orientation preservation of $T_{\bar{\theta}}$.

(d) Flow dynamics via the Poincaré map. In studying the motion of fluid particles the concepts of streamlines, pathlines, and streaklines are very natural. However, as mentioned earlier, their use in the study of unsteady flows is limited since their relationship to such dynamical phenomena as mixing and transport properties may be unclear (Ottino 1988). In fact these concepts may be misleading. For example, Hama (1962) showed that streaklines and pathlines in a time-dependent laminar flow may look turbulent. In the context of the Poincaré map, the dynamical evolution of fluid particles is expressed in terms of the orbits of the Poincare map. The orbit of a fluid particle is defined as follows: Let $p$ be a fluid particle, then the orbit of $p$ under $T$ is the bi-infinite sequence of points given by

$$
\left\{\ldots, T^{-n}(p), \ldots, T^{-1}(p), p, T(p), \ldots, T^{n}(p), \ldots\right\},
$$

where

$n$ factors

$$
\left.T^{n}(p)=T(T, \ldots,(T(p)) \ldots)\right)
$$

\section{REFERENCES}

Annol'd, V. I. 1965 Sur la topologie des ecoulements stationnaires de fluides parfaits. C.R. Acad. Sci. Paris 261, 17.

Arnol'd V. I. 1973 Ordinary Differential Equations. M.I.T. Press.

ARNoL'D V. I. 1978 Mathematical Methods of Classical Mechanics. Springer.

ARnoL'D V. I. 1982 Geometrical Methods in the Theory of Ordinary Differential Equations. Springer. Arnol'd, V. I. \& Avez, A. 1968 Ergodic Problems of Classical Mechanics. W. A. Benjamin.

Arnol'd, V. I. \& Konkine, E. I. 1983 The growth of a magnetic field in a steady compressible flow. Vestn. Mosk. Univ. Mat. Mekh. 3, 43 (in Russian).

AREF, H. 1983 Integrable, chaotic and turbulent vortex motion in two-dimensional flows. Ann. Rev. Fluid Mech. 15, 345.

AREF, H. 1984 Stirring by chaotic advection. J. Fluid Mech. 143, 1.

Aref, H. \& Balachandar, S. 1986 Chaotic advection in a Stokes flow. Phys. Fluids 29, 3515.

AREF, H. \& KAMBE, T. 1988 Report on the IUTAM Symposium : fundamental aspects of vortex motion. J. Fluid Mech. 190, 571.

ARTer, W. 1983 Ergodic streamlines in steady convection. Phys. Lett. 97A, 171.

AUbry, S. \& Le Daeron, P. R. 1983 The discrete Frenkel-Kontorova model and its extensions. 1, Exact results for the ground states. Physica 8D, 381.

BROADWELL, J. E. 1989 A model for reactions in turbulent jets: effects of Reynolds, Schmidt, and Damkohler numbers. In Turbulent Reactive Flows. Lecture Notes in Engineering, Vol. 40 (ed. R. Borghi \& S. N. B. Murthy), pp. 251, Springer. 
Broomhead, D. S. \& Ryrie, S. C. 1988 Particle paths in wavy vortices. Nonlinearity 1, 409.

Chanken, J., Chevray, R., Tabor, M. \& Tan, Q. M. 1986 Experimental study of Lagrangian turbulence in Stokes flow. Proc. R. Soc. London A 408, 105.

Chatken, J., Chu, C. K., TAbor, M. \& TAN, Q. M. 1987 Lagrangian turbulence and spatial complexity in Stokes flow. Phys. Fluids 30,687.

Chien, W.-L, Risina, H. \& Otrino, J. M. 1986 Laminar and chaotic mixing in several cavity flows. J. Fluid Mech. 170, 355.

Danckwerts, P. V. 1953 Continuous flow systems of residence times, Chem. Engng Sci. 2, 1.

Drmotakis, P. E. 1989 Turbulent shear layer mixing with fast chemical reactions. In Turbulent Reactive Flows. Lecture Notes in Engineering, Vol. 40 (ed. R. Borghi \& S. N. B. Murthy), p. 417, Springer.

Dombre, T., Frisch, U., Greene, J. M., Hénon, M., Mehr, A. \& Soward, A. 1986 Chaotic streamlines in the ABC flows. J. Fluid Mech. 167, 353.

Dyson, F. W. 1893 The potential of an anchor ring-Part II. Phil. Trans. R. Soc. Lond. A 184, 1041.

Feingold, M., Kadanoff, L. P. \& Piro, O. 1988 Passive scalars, 3D volume preserving maps and chaos. J. Statist. Phys. 50, 529 .

Fenichel, N. 1971 Persistence and smoothness of invariant manifolds for flows. Indiana Univ. Math. J. 21, 193.

Galloway, D. \& Frisch, U. 1986 Dynamo action in a family of flows with chaotic streamlines. Geophys. Astrophys. Fluid Dyn. 36, 53.

Goldhirsch, I., Sulem, P. L. \& Orszaq, S. A. 1987 Stability and Lyapunov stability of dynamical systems: a different approach and a numerical method. Physica 27D, 311.

Guckenhermer, J. \& Holmes, P. 1983 Non-Linear Oscillations, Dynamical Systems and Bifurcations of Vector Fields. Springer.

HAMA, F. R. 1962 Streaklines in a perturbed shear flow. Phys. Fluids 5, 644.

Hénon, M. 1966 Sur la topologie des lignes de courant dans un cas particular. C. R. Acad. Sci. Paris A 262, 312.

HrRsch, M. W., Puah, C. C. \& Shu B, M. 1977 Invariant Manifolds. Lecture Notes in Mathematics, vol. 583, Springer.

IRwIN, M. C. 1980 Smooth Dynamical Systems. Academic.

KERR, R. M. 1985 Higher-order derivative correlations and the alignment of small-scale structures in isotropic numerical turbulence. J. Fluid Mech. 153, 31.

Kerstein, A. R. \& AshURST, W. T. 1984 Lognormality of gradients of diffusive scalars in homogeneous, two dimensional mixing systems. Phys. Fluids 27, 2819.

KhakhaR, D. V., Rising, H. \& Ottino, J. M. 1986 Analysis of chaotic mixing in two model systems. J. Fluid Mech. 172, 419.

MacKay, R. S., Meiss, J. D. \& Percival, I. C. 1984 Transport in Hamiltonian systems, Physica D 13, 55.

MARBLE, F. E. 1985 Growth of a diffusion flame in the field of a vortex. In Recent Advances in the Aerospace Sciences (ed. C. Casci), pp. 395, Plenum.

Mather, J. N. 1984 Non-existence of invariant circles. Ergod. Theory Dyn. Syst. 4, 301.

Melnikov, V. K. 1963 On the stability of the center for time periodic perturbations. Trans. Moscow Math. Soc. 12, 1.

Moffatt, H. K. \& Proctor, M. R. E. 1985 Topological constraints associated with fast dynamo action. J. Fluid Mech. 154, 493.

Moser, J. 1973 Stable and Random Motions in Dynamical Systems. Princeton University Press.

Otrino, J. M. 1988 The Kinematics of Mixing: Stretching, Chaos, and Transport. Cambridge University Press.

Ottino, J. M., Leong, C. W., Rising, H. \& Swanson, P. D. 1988 Morphological structures produced by mixing in chaotic flows. Nature 333, 419.

Percival, I. C. 1980 Variational principles for invariant tori and cantori. AIP Conf. Proc. Nonlinear Dynamics and the Beam-Beam Interaction, Vol. 57 (ed. M. Mouth \& J. C. Herrera). New York: Am. Inst. Phys. 
Pope, S. B. 1987 Turbulent premixed flames. Ann. Rev. Fluid Mech. 19, 237.

RaLPH, M. E. 1986 Oscillatory flows in wavy-walled tubes, J. Fluid Mech. 168, 515.

Rom-KeDAR, V. 1988 Part I: An analytical study of transport, mixing and chaos in an unsteady vortical flow. Part II : Transport in two dimensional maps. Ph.D. thesis, California Institute of Technology, Pasadena, CA.

Rom-Kedar, V. \& Wiggins, S. 1989 Transport in two-dimensional maps. Arch. Rat. Mech. Anal. (to appear).

Shariff, K. 1989 Dynamics of a class of vortex rings. Ph.D thesis, Dept. of Mech. Engng, Stanford University.

Shariff, K., Leonard, A., Zabusky, N. J. \& Ferziger, J. H. 1988 Acoustics and dynamics of coaxial interacting vortex rings. Fluid Dyn. Res. 3, 337.

SoBEY, I. J. 1985 Dispersion caused by separation during oscillatory flow through a furrowed channel, Chem. Engng Sci. 40, 2129.

Suresh, A. 1985 Point vortex interactions, Ph.D thesis, Princeton University.

Wigains, S. 1987 Chaos in the quasiperiodically forced duffing oscillator. Phys. Lett, 124A, 138.

Wiggins, S. 1988 Global Bifurcations and Chaos-Analytical Methods, Springer.

Yamada, H. \& Matsui, T. 1978 In An Album of Fluid Motion (ed. M. Van Dyke), p. 46. Parabolic. 